\title{
Segmenting Oil Spills from Blurry Images Based on Alternating Direction Method of Multipliers
}

\author{
Fang Chen, Huiyu Zhou, Christos Grecos, Senior Member, IEEE, and Peng Ren, Senior Member, IEEE
}

\begin{abstract}
We exploit the alternating direction method of multipliers (ADMM) for developing an oil spill segmentation method, which effectively detects oil spill regions in blurry synthetic aperture radar (SAR) images. We commence by constructing energy functionals for SAR image deblurring and oil spill segmentation separately. We then integrate the two energy functionals into one overall energy functional subject to a linear mapping constraint that correlates the deblurred image and the segmentation indicator. The overall energy functional along with the linear constraint follows the form of alternating direction method of multipliers and thus enables an effective augmented Lagrangian optimization. Furthermore, the iterative updates in the ADMM maintain information exchanges between the energy minimizations for SAR image deblurring and oil spill segmentation. Most existing blurry image segmentation strategies tend to consider deblurring and segmentation as two independent procedures with no interactions, and the operation of deblurring is thus not guided for obtaining accurate segmentation. In contrast, we integrate deblurring and segmentation into one overall energy minimization framework with information exchanges between the two procedures. Therefore, the deblurring procedure is inclined to operate in favor of more accurate oil spill segmentation. Experimental evaluations validate that our framework outperforms the separate deblurring and segmentation strategy for detecting oil spill regions in blurry SAR images.
\end{abstract}

Index Terms-Deblurring, oil spill segmentation, alternating direction method of multipliers.

\section{INTRODUCTION}

$\mathbf{M}$ ARINE oil spill accidents, which have caused various damages to the natural environment, have frequently occurred at different scales [1]. Satellite-based synthetic aperture radar (SAR) provides an important means for monitoring marine oil spills [2][3] because it has the advantage of allweather and all-time observation ability. In order to make timely damage assessment [4] and spread control of oil spills, it is vital to accurately observe the oil spills through SAR images. Therefore, developing intelligent algorithms for segmenting marine oil spill regions from SAR images has been an important research topic in the field of ocean remote sensing.

This work was supported in part by the National Natural Science Foundation of China under Project 61671481, in part by the Qingdao Applied Fundamental Research under Project 16-5-1-11-jch, in part by the Fundamental Research Funds for the Central Universities under Project 18CX05014A, in part by UK EPSRC under Grants EP/N508664/1, EP/R007187/1 and EP/N011074/1, and in part by Royal Society-Newton Advanced Fellowship under Grant NA160342. (Corresponding Author: Peng Ren)

Fang Chen and Peng Ren are with College of Information and Control Engineering, China University of Petroleum (East China), China (e-mail: chenfangupc@163.com; pengren@upc.edu.cn)

Huiyu Zhou is with Department of Informatics, University of Leicester, United Kingdom (e-mail: hz143@leicester.ac.uk).

Christos Grecos is with the Computer Science Department, Central Washington University, United States (e-mail: grecoschristos@gmail.com).
Most researchers conduct oil spill studies based on SAR data [5] in terms of investigating oil spill physic characteristics for reflecting different types of electromagnetic waves. Here the non-Bragg scattering phenomenon caused by oil spills is a major physical feature for oil spill analysis based on SAR images. The capillary and short gravity waves on the ocean surface give rise to Bragg scattering that is sensed by SAR, and marine oil spills result in dark patches in SAR images by damping out the Bragg scattering. The non-Bragg scattering regions provide indications for observing oil spills based on SAR images. Recently, the more sophisticated polarimetric characteristics of oil spills have been comprehensively investigated and representative state of the art studies include those conducted by Migliaccio et al. [6][7], Ricci et al. [8][9], Minchew et al. [10][11] and Brekke et al. [12][13]. The polarimetry based strategies enhance oil spill observations in SAR images such that basic image processing techniques such as thresholding [14] and $K$-means clustering [7] can be easily applied to detect oil spills in the enhanced representations.

On the other hand, researchers mainly from the image processing community have been working on developing more sophisticated oil spill segmentation methods for accurately detecting oil spill regions in SAR images. In this scenario, one common formulation of oil spill segmentation is energy minimization, where an energy functional measures the segmentation characteristics such as fitness and similarity with respect to the oil spills. Xia et al. [15] developed a continuous energy functional for multiscale nonlocal characterization and employed level set evolution for detecting oil spill contours. Mdakane et al. [16] incorporated a region-based signed pressure force functional into the level set continuous energy functional for detecting oil slicks from moving vessels. Ren et al. [17] proposed to smooth both image and cost volume for discrete energy functions, and apply the graph cut algorithm for obtaining optimal segmentation. Recently, deep learning strategies such as convolutional neural nets [18] and generative adversarial nets [19] have been investigated for oil spill segmentation. These methods normally require a set of segmented images for training a segmentation model. However, the availability of marine oil spill images is not as easy as that of normal images. Such shortage of data becomes even more critical for segmenting blurry marine oil spill images. To train a model addressing the blurry marine oil spill segmentation, a large number of correctly segmented blurry images are required. However, as oil spill accidents are not always observed in blurry conditions, the access to such qualified training data is limited. On the other hand, we directly conduct segmentation on investigated images by ex- 
ploiting energy minimization strategies, which do not require the training procedure. It is thus much more straightforward for practical operations.

Most energy minimization methods perform oil spill segmentation on clear images and tend to ignore the situation that a SAR image is blurred. The SAR imaging is affected by environmental factors such as atmospheric turbulence [20] and inaccurate estimation of the speed of the airborne devices [21] for monitoring ocean. Therefore, blurs are regular phenomena arising in SAR images and pose inevitable interferences to oil spill segmentation.

One possible way to segment oil spills from a blurry SAR image is to conduct deblurring to the blurry image and then perform segmentation on the deblurred image [22]. The flowchart for this separate two-step strategy is illustrated in Fig.1. The two-step strategy is easy to implement. However, most existing deblurring methods [23][24] tend to straightforwardly restore a clear image and do not necessary enhance the effect of the subsequent procedures such as segmentation. One classical method for deblurring images is deconvolution. Recently, a number of new deconvolution strategies [25][26] have been developed for achieving visually higher quality of the restored images. Though these methods improve the visual quality of the restored images, they do not necessarily enhance information required by the subsequent procedures such as segmentation. In order to address this shortcoming, we formulate the problem of SAR image deblurring and oil spill segmentation into one overall energy minimization framework. We present a novel oil spill segmentation framework in which the deblurring and segmentation comprehensively interact with each other. In this scenario, our framework does not consider the deblurring and segmentation as two separate operations but encourages the deblurring to operate in a way that favors accurate segmentation. Therefore, in our framework, the deblurring and segmentation operate in a complementary reciprocal manner such that their intrinsically combined effort essentially improves the effectiveness of oil spill segmentation over the separate deblurring and segmentation strategy.

Our novel energy minimization framework for oil spill segmentation is summarized as follows. We first establish energy functionals for deblurring and oil spill segmentation separately. We then integrate the two energy functionals into one overall energy functional subject to one linear mapping constraint between the deblurred image and the segmentation indicator. The linear mapping not only conveys information between the deblurring and segmentation operations but also enables minimization of the overall energy functional in terms of alternating direction method of multipliers (ADMM) [27][28]. The ADMM decomposes one original problem into several subproblems using the separability of the overall energy functional such that each subproblem is easier to handle. On the other hand, it maintains information conveyed between the subproblems such that the subproblem solutions are compatible with one another and achieve the final solution of the overall energy minimization. We exploit this advantage of ADMM for conducting deblurring which provides helpful information for oil spill segmentation, and thus establish a framework that simultaneously deblurs and segments oil spill
SAR images. Experimental evaluation validates the effectiveness of our method for segmenting blurry oil spill SAR images.

\section{Energy Functional For Deblurring MARine OIL SPILL IMAGES}

This section reviews the energy functional for neutralizing blurs in oil spill images. A deconvolution energy term for restoring images and a regularization term for smoothing kernels are developed separately, and then integrated for penalizing the process of deblurring.

The deblurring scheme presented in this section is a commonly used traditional strategy [29][30]. Based on this existing scheme, we will propose a novel segmentation framework that intrinsically guides the traditional deblurring scheme to operate in favor of accurate segmentation, which will be presented in Section IV.

\section{A. Energy Term for Deconvolution}

Suppose a blurry SAR image $I$ for observing oil spills is formed in terms of the convolution between an original image $I_{d}$ and an unknown blurring kernel $K_{b}$, along with additive Gaussian white noise $n$, as follows:

$$
\begin{aligned}
I(x, y) & =I_{d}(x, y) * K_{b}(x, y)+n(x, y) \\
& =\iint I_{d}(x-u, y-v) K_{b}(u, v) d u d v+n(x, y)
\end{aligned}
$$

where $(x, y)$ denotes one pixel location, $I_{d}(x, y) \geq 0$, $K_{b}(x, y) \geq 0$ and $*$ represents the operation of convolution.

The energy term that characterizes the disagreement between the deblurred image $I_{d}$ and the blurry image $I$ is given as follows:

$$
E_{B}\left(I_{d}, K_{b}\right)=\iint\left[I_{d}(x, y) * K_{b}(x, y)-I(x, y)\right]^{2} d x d y
$$

The blurring kernel $K_{b}$ in (2) is considered as a parameter to be estimated for precisely characterizing the disagreement between the deblurred image $I_{d}$ and the blurry image $I$. The estimation of $K_{b}$ will be described in Section IV. Minimizing the energy term $E_{B}$ in (2) with respect to $I_{d}$ and $K_{b}$ results in deconvolution of these two components from the blurry image $I$. The energy term (2) thus restores the deblurred image $I_{d}$ by maximally neutralizing the interferences from the blurry kernel and the white Gaussian noise on $I$.

\section{B. Kernel Smoothing Regularization}

The blurring kernel $K_{b}$ may contain unexpected abrupt changes especially when it is estimated from a noisy blurry image $I$. To address this drawback, we exploit a smoothing regularization term with respect to $K_{b}$ as follows:

$$
R_{K}\left(K_{b}\right)=\iint\left|\nabla K_{b}(x, y)\right| d x d y
$$

The gradient $\nabla$ characterizes the oil and background variations over the image domain. The regularization term (3) penalizes abrupt changes in $K_{b}$ and thus avoids restoring an image with spikes. 


\section{Energy Functional for Deblurring Oil Spills}

We integrate the deconvolution energy term introduced in Section II-A and the smoothing regularization terms introduced in Section II-B into the energy functional $E_{D}\left(I_{d}, K_{b}\right)$ for deblurring oil spills images, which is given as follows:

$$
E_{D}\left(I_{d}, K_{b}\right)=E_{B}\left(I_{d}, K_{b}\right)+\eta R_{K}\left(K_{b}\right)
$$

where $\eta$ is a positive regularization parameter, which characterizes the trade-off between deconvolution and regularization.

\section{Energy Functional for Segmenting OIL SPILl REGIONS}

This section introduces the energy functional for segmenting oil spills in SAR images. A level set energy term for measuring oil spill fitness, and regularization terms with respect to oil contour length, update regularity and oil edge preservation are developed separately. They are then integrated for characterizing the oil spill segmentation.

Specifically, we not only review the existing region scalable fitting (RSF) segmentation method [31] but also develop a novel oil spill edge preserving regularization term. The new term smooths images without heavily degenerating oil spill edges and thus improves the detail segmentation over the existing RSF method.

\section{A. Level Set Energy Term for Fitness}

We use level sets for detecting oil spills in SAR images [32]. A level set function $\phi$ manifests itself as a surface in a three dimensional space. It is one key optimization parameter for indicating the marine oil spill segmentation in our framework. Specifically, $\phi(x, y) \geq 0$ indicates that the pixel $I_{d}(x, y)$ is segmented into the marine oil spill region, otherwise it is segmented into the background.

We exploit the region-scalable fitting (RSF) level set for characterizing the data fitting in oil spill SAR images. The RSF energy functional is defined as follows:

$$
\begin{aligned}
& E_{F}\left(\phi, r_{1}, r_{2}\right) \\
& =\sum_{i=1}^{2} \alpha_{i} \iint K_{\sigma}(u-x, v-y)\left|I_{d}(u, v)-r_{i}(x, y)\right|^{2} \\
& \quad M_{i}^{\epsilon}(\phi(u, v)) d u d v
\end{aligned}
$$

where $\alpha_{1}$ and $\alpha_{2}$ are positive balancing constants, separately. $I_{d}$ is the deblurred image, and $r_{i}(x, y)$ characterizes the weighted averages of the intensities in a neighborhood of $(\mathrm{x}, \mathrm{y})$ and it represents the image approximation whose update scheme will be introduced in Section IV-D. $M_{1}^{\epsilon}(\phi)=H_{\epsilon}(\phi)$ and $M_{2}^{\epsilon}(\phi)=1-H_{\epsilon}(\phi)$, and $H_{\epsilon}(\phi)$ is the Heaviside function given as follows:

$$
H_{\epsilon}(\phi)=\frac{1}{2}\left[1+\frac{2}{\pi} \arctan \left(\frac{\phi}{\epsilon}\right)\right]
$$

where $\epsilon$ is a positive smooth parameter. The integrations in (5) operate over the spatial domain of the whole image. $M_{i}^{\epsilon}$ controls the integration domain in which the nonnegative Gaussian kernel $K_{\sigma}$ emphasizes the central fitness. Specifically, the integration centered by $r_{i}(x, y)$ just takes place within the $\phi \geq 0$ image region if $i=1$ or within the $\phi<0$ image region if $i=2$. Therefore, $E_{F}$ measures the fitness between the oil spills and the image approximation within the scalable region controlled by $M_{i}^{\epsilon}$.

\section{B. Oil Contour Length Regularization}

The curve obtained in terms of the intersection between $\phi$ and the zero plane (i.e. $\phi(x, y)=0$ ) is referred to as the zero level set of $\phi(x, y)$, and it indicates the contour of an oil spill region. The length of the oil spill contours is approximated as follows:

$$
R_{H_{\epsilon}}(\phi)=\iint\left|\nabla H_{\epsilon}(\phi(x, y))\right| d x d y
$$

It is a commonly used regularization term for level set based segmentation.

\section{Update Regularity Regularization}

Oil spills always exhibit irregular shapes. However, to achieve optimal segmentation, the iterative evolution of $\phi$ for optimization is supposed to be updated in a regular way. To preserve the regularity for updating the level set $\phi$, which is necessary for accurate computation and stable level set evolution, a distance regularization should be intrinsically incorporated into the variational level set energy formulation to maintain the regularity of the level set functional during the evolution [33]. The distance regularization is defined with a potential function as follows:

$$
R_{P}(\phi)=\iint \frac{1}{2}(|\nabla \phi(x, y)|-1)^{2} d x d y
$$

The regularization term $R_{P}(\phi)$ characterizes the deviation of the level set $\phi$ from a signed distance function and also smooths the level set function $\phi$.

\section{Oil Edge Preserving Regularization}

Oil spill edges provide important information for accurately characterizing oil spill regions . However, most existing image denoising methods smooth an image with the trade-off of degenerating oil spill edges. To address this shortcoming, following our preliminary work in [34], we incorporate one recently developed edge preserving filtering technology, i.e. guided filtering [35], into our framework.

In order to have our SAR image smoothed by the guided filter, in our work, we use $I_{d}$ as both the input and guided images, we commence by computing the local features for $I_{d}$. Let $\omega_{(x, y)}$ denote a local square window around $(x, y)$ in $I_{d}$ and $\left|\omega_{(x, y)}\right|$ is the number of pixels in $\omega_{(x, y)}$. The local mean $\mu_{(x, y)}$ and local variance $\sigma_{(x, y)}^{2}$ of $I_{d}$ within $\omega_{(x, y)}$ are given as follows:

$$
\mu(x, y)=\frac{1}{\left|\omega_{(x, y)}\right|} \sum_{(u, v) \in \omega_{(x, y)}} I_{d}(u, v)
$$




$$
\sigma^{2}(x, y)=\frac{1}{\left|\omega_{(x, y)}\right|} \sum_{(u, v) \in \omega_{(x, y)}}\left[I_{d}(u, v)-\mu(x, y)\right]^{2}
$$

The intermediate coefficients $p(x, y)$ and $q(x, y)$ are computed, respectively, as follows:

$$
\begin{gathered}
p(x, y)=\frac{\frac{1}{\left|\omega_{(x, y)}\right|} \sum_{(u, v) \in \omega_{(x, y)}}\left|I_{d}(u, v)\right|^{2}-\left|\mu_{(x, y)}\right|^{2}}{\sigma_{(x, y)}^{2}+\epsilon} \\
q(x, y)=\mu_{(x, y)}-p(x, y) \mu_{(x, y)}
\end{gathered}
$$

where $\epsilon$ is a regularization parameter preventing $p(x, y)$ from being too large.

The mean maps $\bar{p}$ and $\bar{q}$ for $p$ and $q$ are computed as follows:

$$
\begin{aligned}
& \bar{p}(x, y)=\frac{1}{\left|\omega_{(x, y)}\right|} \sum_{(u, v) \in \omega_{(x, y)}} p(u, v) \\
& \bar{q}(x, y)=\frac{1}{\left|\omega_{(x, y)}\right|} \sum_{(u, v) \in \omega_{(x, y)}} q(u, v)
\end{aligned}
$$

Finally, one filtered image pixel $\hat{I}_{d}(x, y)$ is given by

$$
\hat{I}_{d}(x, y)=\bar{p}(x, y) I_{d}(x, y)+\bar{q}(x, y)
$$

We incorporate the guided filter into the RSF energy functional by introducing the following regularization term:

$$
\begin{aligned}
R_{G}(\phi) & =\tau_{1} \iint\left|\nabla \hat{I}_{d}(x, y)\right| H_{\epsilon}(\phi(x, y)) d x d y \\
& +\tau_{2} \iint \hat{I}_{d}(x, y)\left|\nabla H_{\epsilon}(\phi(x, y))\right| d x d y
\end{aligned}
$$

where $\tau_{1}$ and $\tau_{2}$ are positive constants, which balance the two terms for edge preserving regularization.

$\nabla \hat{I}_{d}$ and $\nabla H_{\epsilon}(\phi(x, y))$ characterize the oil spill edges in the smoothed image domain and the level set domain, respectively. In contrast to the gradient of the original $\nabla I_{d}$ which might misidentify noise as oil spill edges, the guided filtered representation $\nabla \hat{I}_{d}$ reflects more accurate oil spill edges without noisy misidentification.

It is motivated by the edge indicating regularizations in that the integration of the product of one edge indicator and one image presentation from different domains possibly slows down the level set evolution around edges and enables a detail edge characterization. In the light of this observation, the terms in (16) regularize the segmentation energy functional in terms of characterizing edge information in both image and level set domains and favor the preservation of edge details.

\section{E. Energy Functional for Segmenting Oil Spill Regions}

We integrate the fitness energy term introduced in Section III-A and the regularization terms introduced in Sections III-B, III-C and III-D into the energy functional $E_{S}\left(\phi, r_{1}, r_{2}\right)$ for segmenting oil spills in SAR images, which is given as follow:

$E_{S}\left(\phi, r_{1}, r_{2}\right)=E_{F}\left(\phi, r_{1}, r_{2}\right)+\gamma_{1} R_{H_{\epsilon}}(\phi)+\gamma_{2} R_{P}(\phi)+\gamma_{3} R_{G}(\phi)$

where $\gamma_{1}, \gamma_{2}$ and $\gamma_{3}$ are positive balancing parameters.

\section{Segmenting Marine Oil Spills from Blurred \\ IMAGes Via Alternating DiRECTION METHOD OF MULTIPLIERS}

One possible way to detect oil spills in a blurry SAR image is to conduct deblurring to the blurry image and then perform segmentation on the deblurred image [22][36]. The flowchart for this separate two-step strategy is illustrated in Fig.1. The two-step strategy is straightforward to implement. However, as the deblurring and segmentation operations are two independent procedures, they cannot communicate to each other. The deblurring operation tends to straightforwardly restore a clear image without considering enhancing the segmentation accuracy. The segmentation operation just aims to detect oil spill regions subject to data fitness but neglects providing indicative information for effective deblurring. Such separate operations neglect the interaction with each other and do not consider maximizing their representational powers in terms of mutual benefit.

We address this shortcoming by developing a blurry oil spill segmentation method that intrinsically incorporates the deblurring operation. A brief review of our method is illustrated in Fig. 2. In contrast to the separate deblurring and segmentation strategy in Fig. 1, our method performs intrinsic interactions between the deblurring and segmentation and encourages the deblurring to operate in favor of accurate segmentation. Detailed descriptions of our novel framework are presented in the following subsections.

\section{A. Overall Energy Functional for Simultaneously Deblurring and Segmenting Marine Oil Spill Images}

We integrate the separate minimizations of deblurring energy functional $E_{D}\left(I_{d}, K_{b}\right)$ and the oil spill segmentation energy functional $E_{S}\left(\phi, r_{1}, r_{2}\right)$ into the minimization of one overall energy functional as follows:

$$
\begin{aligned}
& \operatorname{minimize} E_{D}\left(I_{d}, K_{b}\right)+E_{S}\left(\phi, r_{1}, r_{2}\right) \\
& \text { subject to } \quad a \phi+b I_{d}=c
\end{aligned}
$$

Although the two separate energy functionals $E_{D}\left(I_{d}, K_{b}\right)$ and $E_{S}\left(\phi, r_{1}, r_{2}\right)$ are summed in (18) which results in an overall energy functional. The coefficients $a$ and $b$ establish a linear mapping that correlates the segmentation indicator level set $\phi$ and the deblurred image $I_{d}$. Here a more sophisticated relationship between $\phi$ and $I_{d}$ might exist. However, we use the linear mapping to characterize the relationship between $\phi$ and $I_{d}$ for two reasons. Firstly, it establishes an informative correlation between the segmentation indicator $\phi$ and the deblurred image $I_{d}$. The linear mapping constraint conveys such indicative information and thus enables the information interaction between the deblurring and segmentation steps. Secondly and more importantly, the linear mapping constraint follows the standard form of the alternating direction method of multipliers (ADMM), which provides a reliable solution for the optimization problem in (18) and (19). 


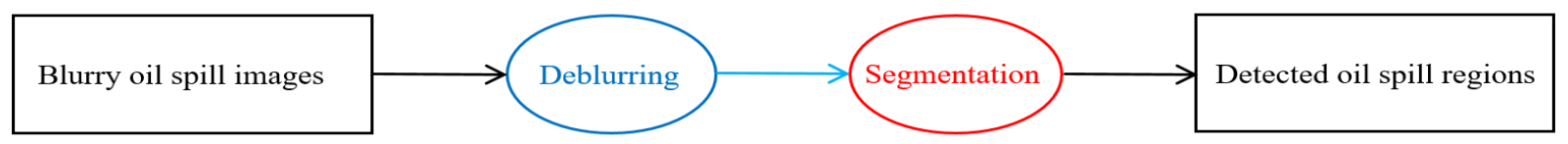

Fig. 1: Separate deblurring and segmentation for detecting oil spills in blurry images.

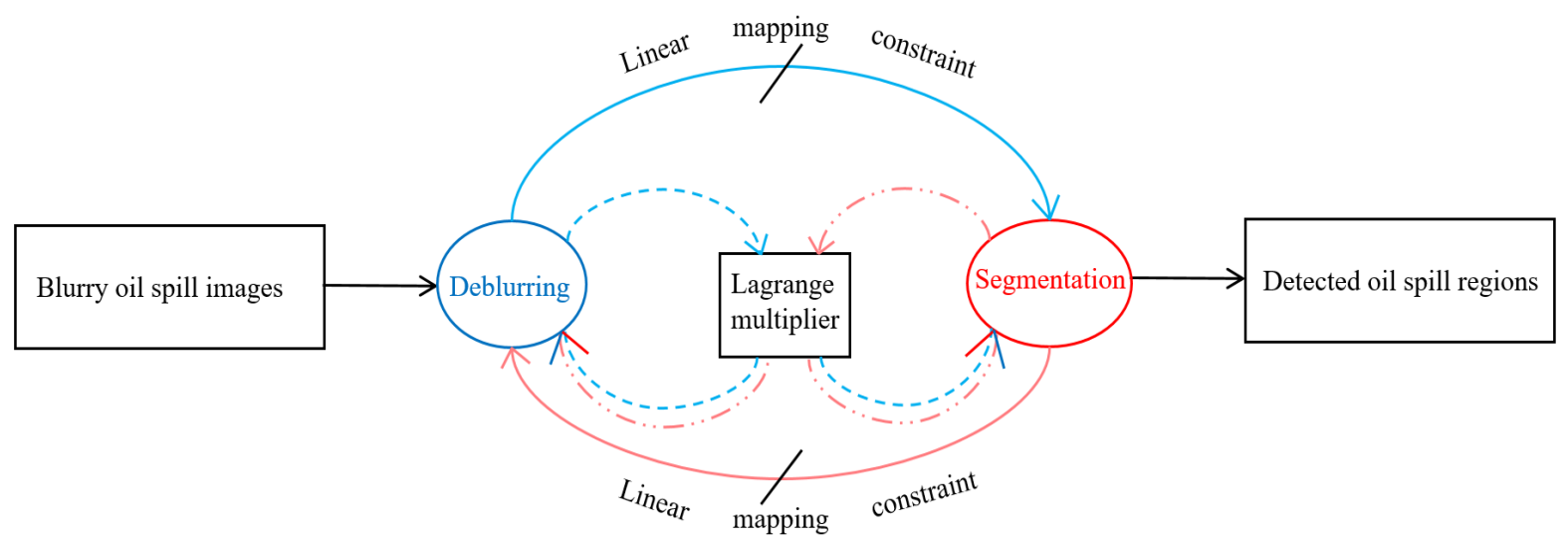

Fig. 2: ADMM based segmentation for detecting oil spills in blurry images.

\section{B. ADMM Form of The Overall Energy Functional}

The alternating direction method of multipliers (ADMM) formulates a constrained optimization problem in terms of the augmented Lagrangian method, which involves a quadratic regularization term. The quadratic regularization relaxes conditions such as differentiability on the energy functional and thus enables more efficient and robust optimization. Detailed introduction to ADMM is referred to [37].

The ADMM form for our overall energy functional shown in (18) and (19) is formulated as follows:

$$
\begin{aligned}
E_{\rho}\left(\phi, I_{d}, l\right)= & E_{S}\left(\phi, r_{1}, r_{2}\right)+E_{D}\left(I_{d}, K_{b}\right)+l^{T}\left(a \phi+b I_{d}-c\right) \\
& +\frac{\rho}{2}\left\|a \phi+b I_{d}-c\right\|_{2}^{2}
\end{aligned}
$$

where $l$ is the Lagrange multiplier to be optimized, and $\rho$ is a positive balancing coefficient. The variable $r_{1}, r_{2}$ and $K_{b}$ are considered as intermediate variables in the ADMM form of the overall energy functional and thus are not global.

ADMM requires two sets of variables. Most existing ADMM strategies set the original optimization variables as one set of ADMM variables and use a set of artificial variables as the other set of ADMM variables. Furthermore, they usually use the linear constraints to enforce the equality of the original variables and the artificial variables. The two sets of variables are then assigned to subproblems intentionally separated from the original energy minimization. Therefore, most existing ADMM methods construct artificial variables and conduct intensional energy separation just for the purpose of efficient computation, and their linear constraints do not reflect practical relationships underlying data representations.
In contrast to the artificial setting of variables, the two major sets of variables in our energy functional (20) are the deblurred image $I_{d}$ and the oil spill segmentation indicating level set $\phi$, both of which are original optimization variables. Furthermore, unlike the intensional energy separation, our energy functional practically consists of the deblurring and segmentation energy functionals, which are naturally separated. However, the separate energy functionals and their variables $I_{d}$ and $\phi$ are correlated by the Lagrangian term $l^{T}\left(a \phi+b I_{d}-c\right)$ and the quadratic regularization term $\frac{\rho}{2}\left\|a \phi+b I_{d}-c\right\|_{2}^{2}$. Therefore, our ADMM form not only enables robust and efficient optimization but also encodes the interaction between SAR image deblurring and oil spill segmentation.

\section{ADMM Minimization of The Overall Energy Functional}

According to the standard ADMM computation, minimization of the ADMM form of the overall energy functional is implemented in terms of iterating the following three basic steps.

In the first step, we fix $\phi$ and $l$, and compute the minimization of the overall energy functional with respect to $I_{d}$ as follows:

$$
I_{d}^{k+1}=\arg \min _{\mathrm{I}_{\mathrm{d}}} E_{\rho}\left(\phi^{k}, I_{d}, l^{k}\right)
$$

where $k$ indicates the iteration number.

In the second step, we fix $I_{d}$ and $l$, and compute minimization of the overall energy functional with respect to $\phi$ as follows:

$$
\phi^{k+1}=\arg \min _{\phi} E_{\rho}\left(\phi, I_{d}^{k+1}, l^{k}\right)
$$


In the third step, we fix $I_{d}$ and $\phi$, and update the Lagrange multiplier $l$ as follows:

$$
l^{k+1}=l^{k}+\rho\left(a \phi^{k+1}+b I_{d}^{k+1}-c\right)
$$

Iteratively implementing the three steps until convergence achieves the minimization of the ADMM form of the overall energy functional. Here the third step plays an extremely important role in our framework. For one thing, it fuses the the information from both the deblurred image $I_{d}^{k+1}$ and the segmentation indicating level set $\phi^{k+1}$. For another, the updated Lagrange multiplier $l^{k+1}$ conveys the fused information of the first and second step for updating the deblurred $I_{d}^{k+1}$ image and the segmentation indicating level set $\phi^{k+1}$ in a new iteration. It is in such iterative way that the information of deblurring and segmentation is exchanged in the process of minimizing the overall energy function.

\section{Energy Minimization Algorithm for Segmenting Oil Spills from Blurry Images}

We describe the exact computation scheme for implementing the three steps presented in Section IV-C.

Step one. In order to obtain optimal $I_{d}^{k+1}$ in the $(k+1)$ th iteration, we commence by computing the intermediate variable $K_{b}^{k+1}$ by solving $\frac{\partial E_{\rho}}{\partial K_{b}}=0$ as follow:

$$
I_{d}^{k} *\left(I_{d}^{k} * K_{b}-I\right)+\eta\left(\nabla \frac{\nabla K_{b}}{\left|\nabla K_{b}\right|}\right)=0
$$

In order to efficiently solve (24) which involves sophisticated operations such as convolution and gradient, we exploit the additive operator splitting method and obtain $K_{b}^{k+1}$ as follows:

$$
K_{b}^{k+1}=\frac{1}{2}\left(K_{b}^{k}\right)^{\frac{1}{4}} \eta\left(K_{b}^{k}+\left(-\tau I_{d}^{k} *\left(I_{d}^{k} * K_{b}^{k}-I\right)\right)\right)
$$

With the blur kernel $K_{b}^{k+1}$ and the other parameters fixed to be the values obtained from the $k$ th iteration, we solve $\frac{\partial E_{\rho}}{\partial I_{d}}=0$ in terms of

$$
\begin{aligned}
& K_{\sigma} *\left[\left(r_{1}\right)^{k} H_{\epsilon}\left(\phi^{k}\right)\right]-I_{d}\left[K_{\sigma} * H_{\epsilon}\left(\phi^{k}\right)\right]+K_{\sigma} *\left\{\left(r_{2}\right)^{k}[\right. \\
& \left.\left.1-H_{\epsilon}\left(\phi^{k}\right)\right]\right\}-I_{d}\left\{K_{\sigma} *\left[1-H_{\epsilon}\left(\phi^{k}\right)\right]\right\}+K_{b}^{k+1} *\left(I_{d} * K_{b}^{k+1}\right. \\
& -I)+\left(l^{k}\right)^{T} b+\rho b\left(a \phi^{k}+b I_{d}-c\right)=0
\end{aligned}
$$

and obtain $I_{d}^{k+1}$ as follows:

$$
\begin{aligned}
I_{d}^{k+1}= & I_{d}^{k}+\tau\left(-K_{\sigma} *\left[\left(r_{1}\right)^{k} H_{\epsilon}\left(\phi^{k}\right)\right]+I_{d}\left[K_{\sigma} * H_{\epsilon}\left(\phi^{k}\right)\right]\right. \\
& -K_{\sigma} *\left\{\left(r_{2}\right)^{k}\left[1-H_{\epsilon}\left(\phi^{k}\right)\right]\right\}+I_{d}\left\{\left(K_{\sigma}\right) *[\right. \\
& \left.\left.1-H_{\epsilon}\left(\phi^{k}\right)\right]\right\}-K_{b}^{k+1} *\left(I_{d} * K_{b}^{k+1}-I\right) \\
& \left.-\left(l^{k}\right)^{T} b-\rho b\left(a \phi^{k}+b I_{d}-c\right)\right)
\end{aligned}
$$

The major computation at this step involves solving the multi-dimensional partial differential equations which requires $O\left(N^{2}\right)$ operations.
Step two. In order to obtain optimal $\phi^{k+1}$ in the $(k+1)$ th iteration, we compute the intermediate variable $r_{i}^{k+1}$ by solving $\frac{\partial E_{\rho}}{\partial r_{i}}=0$ in terms of

$\iint K_{\sigma}(x-u, y-v) M_{i}^{\epsilon}\left(\phi^{k}(u, v)\right)\left(I_{d}^{k+1}(u, v)-r_{i}(x, y)\right) d u v=0$

and obtain $r_{i}^{k+1}$ as follows:

$$
r_{i}^{k+1}=\frac{K_{\sigma} *\left[M_{i}^{\epsilon}\left(\phi^{k}\right) I_{d}^{k+1}\right]}{K_{\sigma} * M_{i}^{\epsilon}\left(\phi^{k}\right)}, i=1,2
$$

With the latest updated intermediate variables $r_{i}^{k+1}$ and the other parameters fixed to be the values obtained from the $k$ th iteration, we have

$$
\begin{aligned}
\frac{\partial E_{\rho}}{\partial \phi}= & -\delta(\phi)\left(\alpha_{1} e_{1}-\alpha_{2} e_{2}\right)+\beta \delta(\phi) \operatorname{div}\left(\frac{\nabla \phi}{|\nabla \phi|}\right) \\
& +\gamma\left(\nabla^{2} \phi-\operatorname{div}\left(\frac{\nabla \phi}{|\nabla \phi|}\right)\right)+\mu_{1} \delta(\phi)\left|\nabla \hat{I}_{d}\right| \\
& +\mu_{2} \hat{I}_{d} \delta(\phi) \operatorname{div}\left(\frac{\nabla \phi}{|\nabla \phi|}\right)+\left(l^{k}\right)^{T} a+\rho a\left(a \phi+b I_{d}^{k+1}-c\right)
\end{aligned}
$$

where $e_{i}$ is given by

$$
\begin{gathered}
e_{i}(x, y)=\iint K_{\sigma}(v-y, u-x)\left|I_{d}^{k+1}(x, y)-\left(r_{i}\right)^{k+1}(u, v)\right|^{2} \\
d u d v, \quad i=1,2
\end{gathered}
$$

and $\delta(\phi)$ is given by

$$
\delta(\phi)=\frac{\partial H_{\epsilon}(\phi)}{\partial \phi}=\frac{1}{\pi} \frac{\epsilon}{\epsilon^{2}+\phi^{2}}
$$

We thus obtain updated $\phi^{k+1}$ as follows:

$$
\phi^{k+1}=\phi^{k}+t \frac{\partial E_{\rho}}{\partial \phi}
$$

where $t$ is a fixed learning rate. The major computation at this step involves the divergence of a gradient which requires $O\left(N^{2}\right)$ operations.

Step three. In the third step, we fix $I_{d}$ and $\phi$, and update the Lagrange multiplier $l$ according to (23). It should be observed from (23) that each updated Lagrange multiplier $l^{k}$ fuses the the information from both the deblurred image $I_{d}^{k}$ and the segmentation indicating level set $\phi^{k}$ in the previous iteration. Additionally, the updated Lagrange multiplier $l^{k}$ conveys itself to the updates of $I_{d}^{k+1}$ in (27) and $\phi^{k+1}$ in (30) in the current iteration. Therefore, the iterative ADMM computation turns out to be a rotating update of the deblurred image $I_{d}$ and the segmentation indicator $\phi$, where the two factors affect each other and the Lagrange multiplier $l$ plays a role of messenger for transferring information between them.

The summarized description of our novel ADMM framework is illustrated in Fig. 2. In contrast to the separate deblurring and segmentation shown in Fig. 1, the ADMM framework maintains rotating information exchanges over iterations. As illustrated in Fig. 2, the information exchanges arise in a twofold manner, i.e. the outer cycle updates subject to the linear 
mapping constraint and the inner interaction via the Lagrange multiplier. Firstly, the deburred image $I_{d}^{k+1}$ is updated with the consideration of the latest segmentation indicator $\phi^{k}$ according to (27); similarly, the segmentation indicator $\phi^{k+1}$ is updated with the consideration of the latest deblurred image $I_{d}^{k+1}$ according to (30) and (33). These mutually affected updates form the outer cycle information exchanges shown in Fig. 2. Secondly, the Lagrange multiplier $l^{k+1}$ fuses information from the latest deblurred image $I_{d}^{k+1}$ and the segmentation indicator $\phi^{k+1}$ according to (23) and conveys itself to the next iteration for updating the new deblurred image according to (27) and the new segmentation indicator according to (30) and (33). These operations regarding information fusion and conveying are depicted by the inner interaction via the Lagrange multiplier shown in Fig. 2. Specifically, two different style single-line arrows point from the deblurring and segmentation boxes to the Lagrange multiplier box separately. Additionally, two double-line arrows point from the Lagrange multiplier box to the deblurring and segmentation boxes separately. The double-line arrows reflect that the Lagrange multiplier fuses information from the deblurring and the segmentation which is delivered by the two different styled single-line arrows. Then the two double-line arrows convey the fused information back to compute the new deblurring and segmentation separately. Therefore, the deblurring and segmentation are not isolated in our ADMM framework but comprehensively interact with each other in a sense of alternating direction. This partially explains why the framework we exploit in our work is referred to as the alternating direction method of multipliers in the literature. The deblurring procedure within the ADMM is guided to restore images in favor of accurate segmentation, and deblurring cues also empower more effective oil spill segmentation.

The algorithm for implementing our framework is illustrated as Algorithm 1. Specifically, we initialize $I_{d}^{0}$ by the original image $I, K_{b}^{0}$ by an all one matrix, and $\phi^{0}$ by a rectangle around one oil spill region.

\section{Algorithm 1 Segmenting Oil Spills from Blurry Images Based on Alternating Direction Method of Multipliers}

Input: An blurry oil spill SAR image $I$.

$I_{d}^{0} \leftarrow I, K_{b}^{0}=\operatorname{ones}(\operatorname{size}(I)), \phi^{0}=$ initialLSF,$l^{0}=\mathbf{1}$.

$K_{\sigma} \leftarrow$ Gaussian kernel, $H_{i}^{\epsilon} \leftarrow$ Heaviside function.

For from $k=1$ until convergence: do

Solve $\frac{\partial E_{\rho}}{\partial K_{b}}=0$, update $K_{b}^{k+1}$ based on (25).

Solve $\frac{\partial E_{\rho}}{\partial I_{d}}=0$, update $I_{d}^{k+1}$ based on (27).

Solve $\frac{\partial E_{\rho}}{\partial r_{i}}=0$, calculate $r_{i}^{k+1}$ based on (29).

Solve $\frac{\partial E_{\rho}}{\partial \phi}=0$, update $\phi^{k+1}$ based on (33).

Solve $\frac{\partial E_{\rho}}{\partial l}=0$, update $l^{k+1}$ based on (23).

\section{End for}

Output: Detected oil spill regions for $I$.

\section{EXPERIMENTAL VALIDATION}

To validate the effectiveness of the proposed ADMM framework, we commence by using the SAR images with VV polarization obtained from the NOWPAP database ${ }^{1}$, which contain differently shaped marine oil spill regions, as our test dataset. The images we used in the experiment are SAR images including C-band SAR images from the ERS-1 and ERS-2 satellites, and C-band ASAR images from the Envisat satellite. These images containing oil spills with various shapes are captured in separate time by different sensors. We describe the information of SAR image sources and sensor properties in Tables I and II, where the symbol "." indicates unavailable information.

TABLE I: NOWPAP image descriptions.

\begin{tabular}{c|c|c}
\hline \hline Capture time & Satellite & Image cover ground \\
\hline $19.06 .199502: 30: 40$ & ERS-1 & $394 \mathrm{~km} 2$ \\
\hline $02.09 .199602: 00: 55$ & ERS-2 & $17.8 \times 106 \mathrm{~m} 2$ \\
\hline $20.07 .199702: 14: 41$ & ERS-2 & - \\
\hline $27.09 .199902: 02: 05$ & ERS-2 & - \\
\hline $27.09 .199902: 02: 35$ & ERS-2 & - \\
\hline $29.05 .200002: 02: 32$ & ERS-2 & - \\
\hline $15.08 .200713: 04: 01$ & Envisat & - \\
\hline $16.08 .200701: 16: 02$ & Envisat & - \\
\hline $01.09 .200801: 11: 51$ & Envisat & - \\
\hline \hline
\end{tabular}

TABLE II: SAR satellite and image descriptions.

\begin{tabular}{c|c|c|c}
\hline \hline Satellite & Spatial resolution & Waveband & Image level \\
\hline ERS-1,2 SAR & $30 \mathrm{~m} \times 30 \mathrm{~m}$ & C-band & 2 \\
\hline Envisat ASAR & $150 \mathrm{~m} \times 150 \mathrm{~m}$ & C-band & 2 \\
\hline \hline
\end{tabular}

We use the region scalable fitness (RSF) level set [31] as a baseline for comparing segmentation accuracy. The RSF level set is one state of the art image segmentation method. We have modified the RSF level set by incorporating the edge preserving regularization and developed our energy functional (20) in Section IV-B. In order to validate the effectiveness of our framework, we conduct experiments for comparison as follows: (a) Applying the RSF level set method directly to blurry images and obtaining the oil spill segmentation results; (b) First performing deblurring by minimizing (4) and then conducting graph cuts, DRLSE (distance regularized level set evolution) or RSF level set segmentation for obtaining the oil spill segmentation results, (c) Operating ADMM for minimizing the proposed overall energy functional for obtaining the oil spill segmentation results. We test different methods subject to imposing Gaussian blur and motion blur on SAR images. However, the blur type is totally blind to the ADMM in the integrated deblurring and segmentation process.

\footnotetext{
${ }^{1}$ http://cearac.poi.dvo.ru/en/db/
} 
Fig. 3 illustrates the visual results for different segmentation strategies subject to the Gaussian blur. Specifically, Fig. 3(a1) illustrates the blurry SAR images which are blurred by an unknown Gaussian. Fig. 3(a-2) illustrates the segmentation results obtained by applying the RSF level set method directly to the blurry images. As the images are not deblurred before segmentation, the oil spill segmentation results thus obtained contain certain blurs with considerable detail loss. Fig. 3(b1) illustrates the deblurred results by just minimizing the deblurring energy functional introduced in Section II-C. Figs. 3(b-2), 7(b-3) and 7(b-4) illustrate the segmentation results by applying the graph cuts, DRLSE and RSF level set method to the deblurred images shown in Fig. 3(b-1). It is clear that these segmentation results preserve more details than those shown in Fig. 3(a-2). This validates that the separate deblurring and segmentation strategy outperforms the straightforward segmentation strategy. However, they still exhibit differences from the ground truth segmentation shown in Fig. 3(d). Fig. 3(c) illustrates the oil spill segmentation results from applying our ADMM framework to the blurry SAR images in Fig. 3(a1). It is clear that our ADMM framework generates the most accurate segmentation results, and outperforms the straightforward RSF level set strategy and the separate deblurring and segmentation strategy. Additionally, the visual experimental results subject to the motion blur are illustrated in Fig. 4, where we observe that our ADMM method also outperforms the alternative comparison methods.

In order to further evaluate the proposed method, in addition to the experiments on the NOWPAP dataset, we test our method on three airborne L-band Uninhabited Aerial Vehicle Synthetic Aperture Radar (UAVSAR) images with HH and VV polarization and a spatial resolution of $6 \mathrm{~m}$. The experimental results are illustrated in Figs. 5 and 6.

Furthermore, we test our method and different comparison methods on MODIS oil spill images and the visual results are given in Figs. 7 and 8. MODIS is not SAR and the experimental evaluations on MODIS images validate that our framework is not only suitable for processing SAR images but also potentially applies to other types of remote sensing images for segmenting oil spills.

To quantitatively evaluate the performance of different segmentation algorithms, we compute the recall ( $\left.\frac{\# \text { correctly segmented pixels }}{\# \text { oil spill pixels }}\right)$ and precision ( $\frac{\# \text { correctly segmented pixels }}{\# \text { segmented pixels }}$ ) for segmentation results of alternative comparison methods. Tables III, IV, VI and V provide the quantitative experimental results. It is clear that the straightforward segmentation without deblurring is the most inferior strategy and our ADMM segmentation achieves the highest accuracy. The quantitative experimental results comply with the visual observations of Figs. 3, 4, 5, 6, 7 and 8 .

Figs. 9 and 10 illustrate the segmentation accuracies in terms of recall and precision for alternative methods over 50 NOWPAP SAR, UAVSAR and MODIS images containing oil spills. Standard deviations are also marked on the top of corresponding accuracy bars. We can see that our ADMM framework outperforms the two comparison methods in terms of both recall and precision with relative small standard deviations.

To make the quantitative evaluation one step further, we compare the convergence rates of different strategies by using the top oil spill UAVSAR image in Fig. 6(a-1). Fig. 11 illustrates the convergence rates of different strategies with respect to iteration numbers. Fig. 12 illustrates the convergence rates of different strategies with respect to runtime under Matlab 2016b implementation based on Intel(R) Core(TM) i5-3470 CPU. We can see that our ADMM method not only produces more accurate segmentation results but also achieves much better convergence rate than the other two comparison strategies. This efficiency benefits from the information exchange between the blurring and segmentation procedures in ADMM, which guides the deblurring to operate in favor of more accurate segmentation and thus enables fast convergence to the minimum energy.

\section{CONCLUSIONS}

We have described how to exploit the alternating direction method of multipliers (ADMM) for accurately segmenting oil spill regions in synthetic aperture radar (SAR), uninhabited aerial vehicle synthetic aperture radar (UAVSAR) and moderate-resolution imaging spectroradiometer (MODIS) blurry images. Existing strategies tend to consider deblurring and segmentation as two separate steps and the deblurring procedure is not guided in favor of accurate segmentation. To address this shortcoming, we have formulated images deblurring and oil spill segmentation in one overall energy functional, and established a linear mapping for characterizing the deblurring and segmentation relationship. We have used ADMM to minimize the overall energy functional. The iterative updates in ADMM not only fuse information both from deblurring and segmentation but also convey the fused information updates for deblurring and segmentation. Therefore, our framework is able to perform effective deblurring in favor of accurate segmentation. Experimental results have validated that our framework outperforms the separate deblurring and segmentation strategy for detecting oil spill regions in blurry NOWPAP SAR, UAVSAR and MODIS images.

\section{REFERENCES}

[1] P. F. Kingston, "Long-term environmental impact of oil spills," Spill Science \& Technology Bulletin, vol. 7, no. 1, pp. 53-61, 2002.

[2] B. Fiscella, A. Giancaspro, F. Nirchio, P. Pavese, and P. Trivero, "Oil spill detection using marine SAR images," International Journal of Remote Sensing, vol. 21, no. 18, pp. 3561-3566, 2000.

[3] A. H. Solberg, C. Brekke, and P. O. Husoy, "Oil spill detection in Radarsat and Envisat SAR images," IEEE Transactions on Geoscience and Remote Sensing, vol. 45, no. 3, pp. 746-755, 2007.

[4] T. Soukissian, F. Karathanasi, and P. Axaopoulos, "Satellite-based offshore wind resource assessment in the Mediterranean sea," IEEE Journal of Oceanic Engineering, vol. 42, no. 1, pp. 73-86, 2017.

[5] D. Velotto, C. Bentes, B. Tings, and S. Lehner, "First comparison of Sentinel-1 and protectTerraSAR-X data in the framework of maritime targets detection: South Italy case," IEEE Journal of Oceanic Engineering, vol. 41, no. 4, pp. 993-1006, 2016.

[6] F. Nunziata, M. Migliaccio, and X. Li, "Sea oil slick observation using hybrid-polarity SAR architecture," IEEE Journal of Oceanic Engineering, vol. 40, no. 2, pp. 426-440, 2015. 
TABLE III: Accuracy of oil spill segmentation for Gaussian blurry NOWPAP SAR images.

\begin{tabular}{|c|c|c|c|c|c|c|}
\hline \multirow{2}{*}{\multicolumn{2}{|c|}{ Method }} & 1 & 2 & 3 & 4 & 5 \\
\hline & & & & & & \\
\hline \multirow{2}{*}{ Straightforward segmentation } & Recall & 0.6919 & 0.7687 & 0.7719 & 0.7833 & 0.7225 \\
\hline & Precision & 0.7366 & 0.8076 & 0.8085 & 0.8127 & 0.7643 \\
\hline \multirow{2}{*}{$\begin{array}{l}\text { Separate deblurring } \\
\text { and segmentation } \\
\text { (graph cuts) }\end{array}$} & Recall & 0.7286 & 0.8329 & 0.8426 & 0.8129 & 0.7396 \\
\hline & Precision & 0.8574 & 0.8627 & 0.9096 & 0.9103 & 0.8735 \\
\hline \multirow{2}{*}{$\begin{array}{l}\text { Separate deblurring } \\
\text { and segmentation } \\
\text { (DRLSE) }\end{array}$} & Recall & 0.7303 & 0.8421 & 0.8501 & 0.8228 & 0.7481 \\
\hline & Precision & 0.8698 & 0.8703 & 0.9128 & 0.9107 & 0.8843 \\
\hline \multirow{2}{*}{$\begin{array}{l}\text { Separate deblurring } \\
\text { and segmentation } \\
\text { (RSF) }\end{array}$} & Recall & 0.7451 & 0.8692 & 0.8587 & 0.8342 & 0.7520 \\
\hline & Precision & 0.8769 & 0.8798 & 0.9179 & 0.9122 & 0.8905 \\
\hline \multirow{2}{*}{ ADMM segmentation } & Recall & 0.8537 & 0.8769 & 0.9105 & 0.8579 & 0.8092 \\
\hline & Precision & 0.9108 & 0.9307 & 0.9578 & 0.9531 & 0.9082 \\
\hline
\end{tabular}

TABLE IV: Accuracy of oil spill segmentation for motion blurry NOWPAP SAR images.

\begin{tabular}{|c|c|c|c|c|c|c|}
\hline \multirow{2}{*}{\multicolumn{2}{|c|}{ Method }} & 1 & 2 & 3 & 4 & 5 \\
\hline & & & & & & \\
\hline \multirow{2}{*}{ Straightforward segmentation } & Recall & 0.7308 & 0.7426 & 0.7721 & 0.7280 & 0.7493 \\
\hline & Precision & 0.8140 & 0.7859 & 0.7148 & 0.8413 & 0.8761 \\
\hline \multirow{2}{*}{$\begin{array}{l}\text { Separate deblurring } \\
\text { and segmentation } \\
\text { (graph cuts) }\end{array}$} & Recall & 0.7869 & 0.7882 & 0.8122 & 0.7309 & 0.7537 \\
\hline & Precision & 0.8331 & 0.8076 & 0.7796 & 0.8549 & 0.8806 \\
\hline \multirow{2}{*}{$\begin{array}{l}\text { Separate deblurring } \\
\text { and segmentation } \\
\text { (DRLSE) }\end{array}$} & Recall & 0.8425 & 0.8079 & 0.8578 & 0.7562 & 0.7623 \\
\hline & Precision & 0.8703 & 0.8189 & 0.8293 & 0.8597 & 0.8903 \\
\hline \multirow{2}{*}{$\begin{array}{l}\text { Separate deblurring } \\
\text { and segmentation } \\
\text { (RSF) }\end{array}$} & Recall & 0.8575 & 0.8163 & 0.8651 & 0.7693 & 0.7648 \\
\hline & Precision & 0.8946 & 0.8392 & 0.8342 & 0.8601 & 0.8917 \\
\hline \multirow{2}{*}{ ADMM segmentation } & Recall & 0.8832 & 0.8717 & 0.8849 & 0.8147 & 0.8964 \\
\hline & Precision & 0.9101 & 0.9174 & 0.9412 & 0.9051 & 0.9182 \\
\hline
\end{tabular}


TABLE V: Accuracy of oil spill segmentation for Gaussian and motion blurry UAVSAR images.

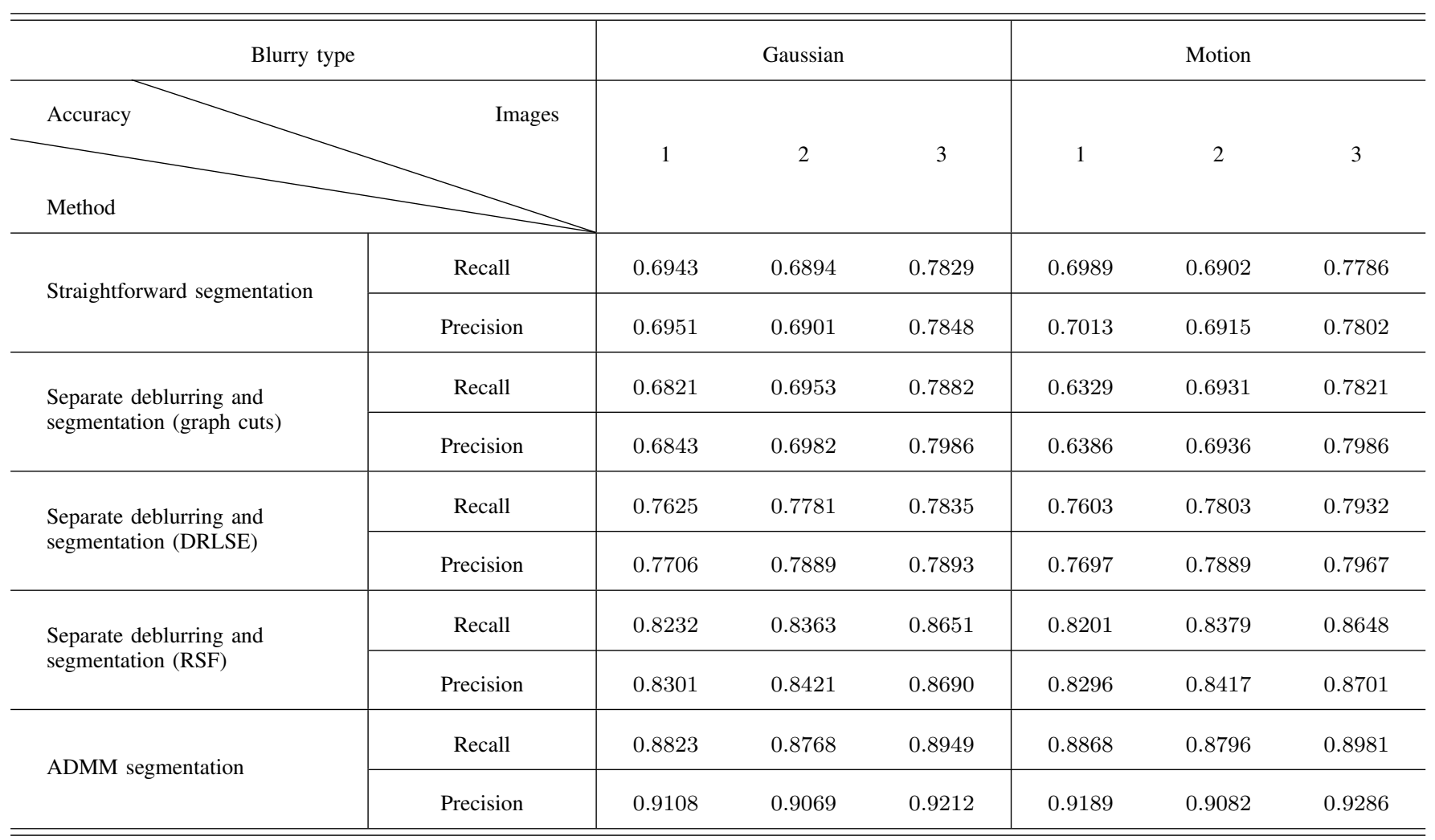

TABLE VI: Accuracy of oil spill segmentation for Gaussian and motion blurry MODIS images.

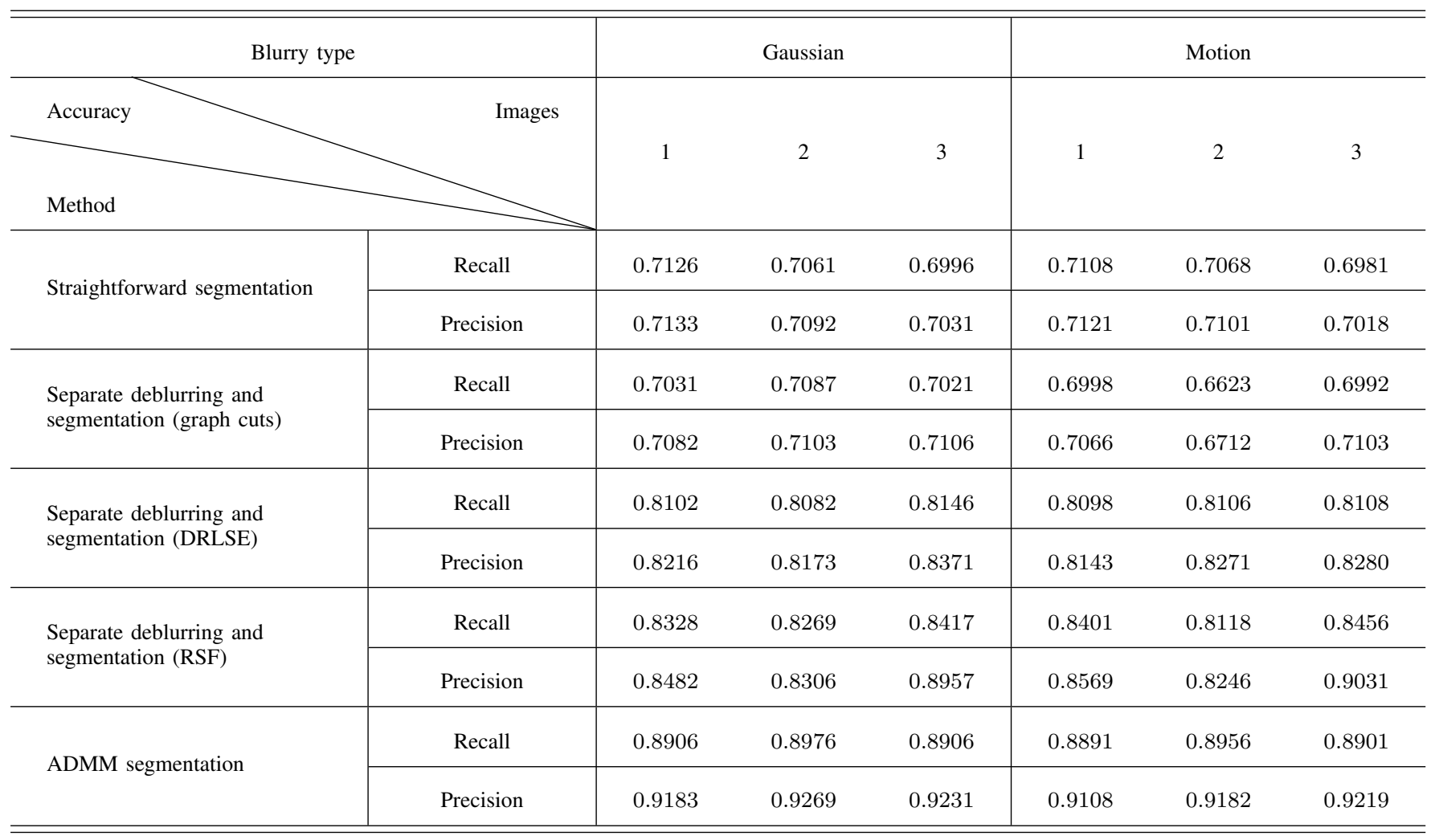


[7] A. Buono, F. Nunziata, M. Migliaccio, and X. Li, "Polarimetric analysis of compact-polarimetry SAR architectures for sea oil slick observation," IEEE Transactions on Geoscience and Remote Sensing, vol. 54, no. 10, pp. 5862-5874, 2016.

[8] F. Bandiera and G. Ricci, "Slicks detection on the sea surface based upon polarimetric SAR data," IEEE Geoscience and Remote Sensing Letters, vol. 2, no. 3, pp. 342-346, 2005.

[9] F. Bandiera, A. Masciullo, and G. Ricci, "A bayesian approach to oil slicks edge detection based on SAR data," IEEE Transactions on Geoscience and Remote Sensing, vol. 52, no. 5, pp. 2901-2909, 2014.

[10] B. Minchew, C. E. Jones, and B. Holt, "Polarimetric analysis of backscatter from the Deepwater Horizon oil spill using L-band synthetic aperture radar," IEEE Transactions on Geoscience and Remote Sensing, vol. 50, no. 10, pp. 3812-3830, 2012.

[11] M. J. Collins, M. Denbina, B. Minchew, C. E. Jones, and B. Holt, "On the use of simulated airborne compact polarimetric SAR for characterizing oil-water mixing of the Deepwater horizon oil spill," IEEE Journal of Selected Topics in Applied Earth Observations and Remote Sensing, vol. 8, no. 3, pp. 1062-1077, 2015.

[12] C. Brekke, C. E. Jones, S. Skrunes, B. Holt, M. Espeseth, and T. Eltoft, "Cross-correlation between polarization channels in sar imagery over oceanographic features," IEEE Geoscience Remote Sensing Letters, vol. 13, no. 7, pp. 997-1001, 2016.

[13] M. M. Espeseth, S. Skrunes, C. E. Jones, C. Brekke, B. Holt, and A. P. Doulgeris, "Analysis of evolving oil spills in full-polarimetric and hybrid-polarity SAR," IEEE Transactions on Geoscience and Remote Sensing, vol. 55, no. 7, pp. 4190-4210, 2017.

[14] A. Lupidi, D. Staglianò, M. Martorella, and F. Berizzi, "Fast detection of oil spills and ships using SAR images," Remote Sensing, vol. 9, no. 3, p. 230, 2017.

[15] G.-S. Xia, G. Liu, W. Yang, and L. Zhang, "Meaningful object segmentation from SAR images via a multiscale nonlocal active contour model," IEEE Transactions on Geoscience and Remote Sensing, vol. 54, no. 3, pp. 1860-1873, 2016.

[16] L. W. Mdakane and W. Kleynhans, "An image-segmentation-based framework to detect oil slicks from moving vessels in the southern african oceans using SAR imagery," IEEE Journal of Selected Topics in Applied Earth Observations and Remote Sensing, vol. 10, no. 36, pp. 2810-2818, 2017.

[17] P. Ren, M. Di, H. Song, C. Luo, and C. Grecos, "Dual smoothing for marine oil spill segmentation," IEEE Geoscience and Remote Sensing Letters, vol. 13, no. 1, pp. 82-86, 2016.

[18] D. Malmgren-Hansen and M. Nobel-JRgensen, "Convolutional neural networks for sar image segmentation," in IEEE International Symposium on Signal Processing and Information Technology, 2015, pp. 231-236.

[19] X. Yu, H. Zhang, C. Luo, H. Qi, and P. Ren, "Oil spill segmentation via adversarial $f$-divergence learning," IEEE Transactions on Geoscience and Remote Sensing, doi:10.1109/TGRS.2018.2803038, 2018.

[20] Y. K. Bi, J. P. Sun, X. Han, and Y. P. Wang, "Effects of atmospheric turbulence on high resolution sar performance," Applied Mechanics Materials, vol. 130-134, pp. 86-89, 2012.

[21] Z. S. Liu, B. N. Wang, M. S. Xiang, and L. Y. Chen, "Performance analysis for airborne interferometric sar affected by flexible baseline oscillation," Journal of Radars, vol. 3, no. 2, 2014.

[22] R. Chan, H. Yang, and T. Zeng, "A two-stage image segmentation method for blurry images with poisson or multiplicative gamma noise," Siam Journal on Imaging Sciences, vol. 7, no. 1, pp. 98-127, 2014

[23] J. Biemond, R. L. Lagendijk, and R. M. Mersereau, "Iterative methods for image deblurring," Proceedings of the IEEE, vol. 78, no. 5, pp. 856883, 2002.

[24] J. P. Oliveira, J. M. Bioucas-Dias, and M. A. T. Figueiredo, "Adaptive total variation image deblurring: A majorizationminimization approach," Signal Processing, vol. 89, no. 9, pp. 1683-1693, 2009.

[25] L. Chen and T. Zeng, "A convex variational model for restoring blurred images with large rician noise," Journal of Mathematical Imaging Vision, vol. 53, no. 1, pp. 92-111, 2015.

[26] T. F. Chan and C. K. Wong, "Total variation blind deconvolution," IEEE Transactions on Image Processing A Publication of the IEEE Signal Processing Society, vol. 7, no. 3, pp. 370-375, 1998.

[27] S. Boyd, N. Parikh, E. Chu, B. Peleato, and J. Eckstein, "Distributed optimization and statistical learning via the alternating direction method of multipliers," Foundations Trends in Machine Learning, vol. 3, no. 1, pp. 1-122, 2011.

[28] M. Hong and Z. Q. Luo, "On the linear convergence of the alternating direction method of multipliers," Mathematical Programming, vol. 162, no. 1-2, pp. 1-35, 2012.
[29] Z. Rti, "Deblurring images blurred by the discrete gaussian," Applied Mathematics Letters, vol. 8, no. 8, pp. 29-35, 1995.

[30] S. H. Lee, H. M. Park, and S. Y. Hwang, "Motion deblurring using edge map with blurred/noisy image pairs," Optics Communications, vol. 285, no. 7, pp. 1777-1786, 2012.

[31] C. Li, C.-Y. Kao, J. C. Gore, and Z. Ding, "Minimization of regionscalable fitting energy for image segmentation," IEEE transactions on image processing, vol. 17, no. 10, pp. 1940-1949, 2008.

[32] K. Karantzalos and D. Argialas, "Automatic detection and tracking of oil spills in sar imagery with level set segmentation," International Journal of Remote Sensing, vol. 29, no. 21, pp. 6281-6296, 2008.

[33] C. Li, C. Xu, C. Gui, and M. D. Fox, "Distance regularized level set evolution and its application to image segmentation," IEEE transactions on image processing, vol. 19, no. 12, pp. 3243-3254, 2010.

[34] F. Chen, X. Yu, X. Jiang, and P. Ren, "Level set with self-guided filtering for marine oil spill segmentation," in Proceedings IEEE International Geoscience and Remote Sensing Symposium, 2017.

[35] K. He, J. Sun, and X. Tang, "Guided image filtering," IEEE Transactions on Pattern Analysis Machine Intelligence, vol. 35, no. 6, pp. 1397-1409, 2013.

[36] H. Wang, T. Z. Huang, Z. Xu, and Y. Wang, "A two-stage image segmentation via global and local region active contours," Neurocomputing, vol. 205, no. C, pp. 130-140, 2016.

[37] W. Bo, S. Boyd, M. Annergren, and Y. Wang, "An admm algorithm for a class of total variation regularized estimation problems *," IFAC Proceedings Volumes, vol. 45, no. 16, pp. 83-88, 2012.

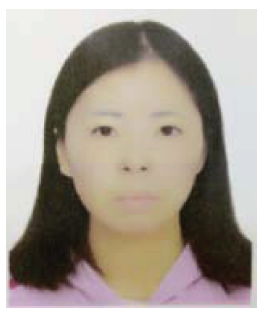

Fang Chen received the B. Eng. degree in Communication Engineering from Southwest University for Nationalities, Chengdu, China, in 2014. She is currently pursuing the M. Eng. degree in Information and Communication engineering in China University of Petroleum (East China), Qingdao, China.

Her current research interests include machine learning and signal processing, with application to remote sensing image classification and segmentation.

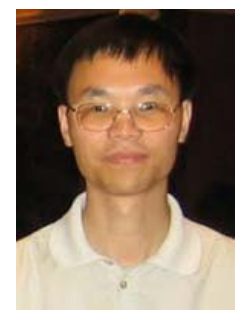

Huiyu Zhou received a B.E. degree in Radio Technology from Huazhong University of Science and Technology of China, and a Master of Science degree in Biomedical Engineering from University of Dundee of UK. He was then awarded the PhD degree in Computer Vision from Heriot-Watt University, UK. Dr. Zhou presently is a Reader at Department of Informatics, University of Leicester, UK. 


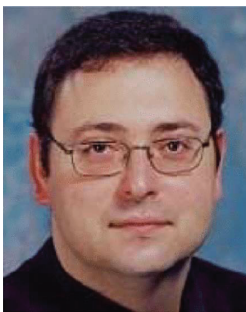

Christos Grecos received a Bachelors degree in Computer Science (Systems Software Option) from Concordia University in Montreal, Canada, a Masters of Science degree in Human Computer Interaction from Heriot Watt University in Edinburgh, Scotland (UK). His PhD degree in Image and Video Coding algorithms for standards is from Glamorgan University (later renamed University of South Wales in Wales, UK). He is currently a tenured full time Professor and Chair of the Computer Science Department of Central Washington University (CWU) in US. His research interests include image/video compression standards, image/video processing and analysis, image/video networking and computer vision.

Dr Grecos has received the best poster award in the 2012 IEEE Conference in Consumer Electronics and the First Place Student paper award in the 2014 IEEE Conference in Consumer Electronics. He has also received the best student paper award in the 2014 International Conference on Signal Processing and Multimedia Applications (SIGMAP) and the best student paper prize in the 2014 Irish Machine Vision and Image Processing Conference.

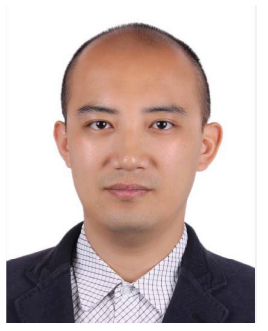

Peng Ren $\left(\mathrm{SM}^{\prime} 16\right)$ received the B.Eng. and M.Eng. degrees in electronic engineering from the Harbin Institute of Technology, Harbin, China, and the Ph.D. degree in computer science from the University of York, York, U.K. He is currently a Professor with the College of Information and Control Engineering, China University of Petroleum (East China), Qingdao, China. His research interests include remote sensing and machine learning.

Dr. Ren was a recipient of the K. M. Scott Prize from the University of York in 2011 and the Eduardo Caianiello Best Student Paper Award at the 18th International Conference on Image Analysis and Processing in 2015, as one co-author. 

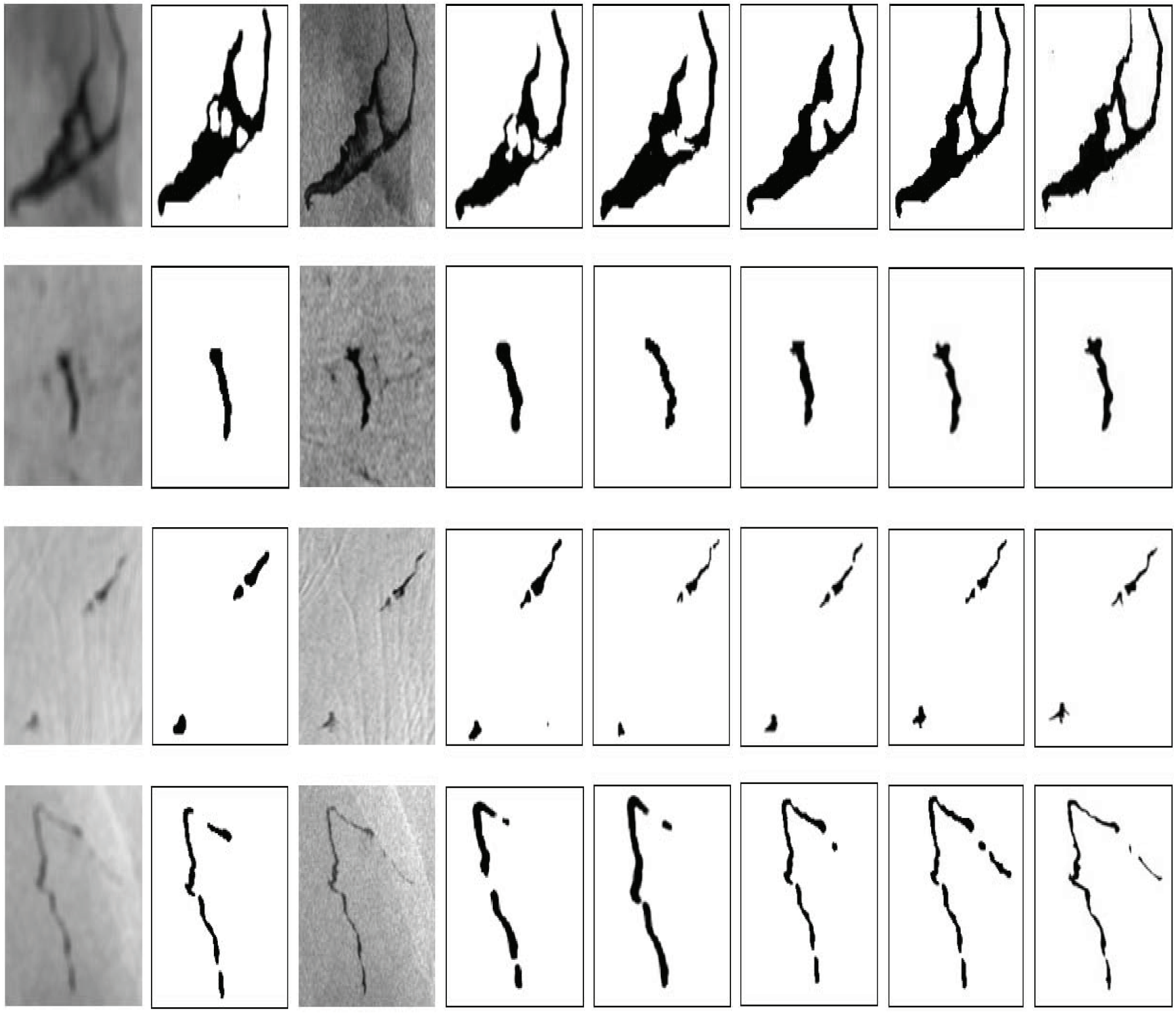

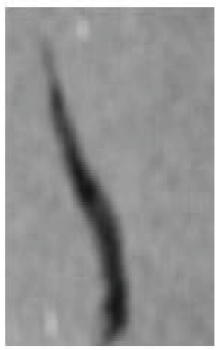

(a-1)

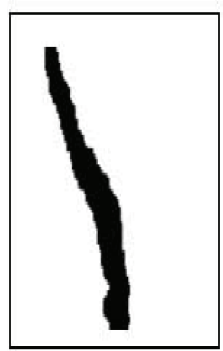

(a-2)

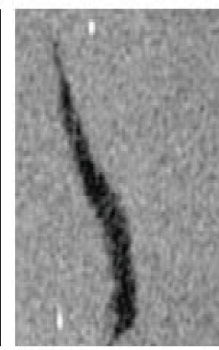

(b-1)

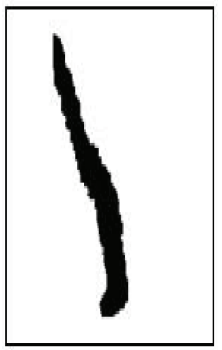

(b-2)

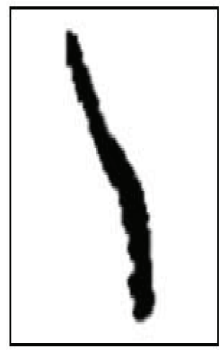

(b-3)

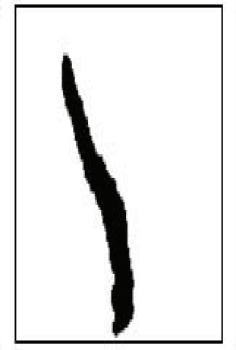

(b-4)

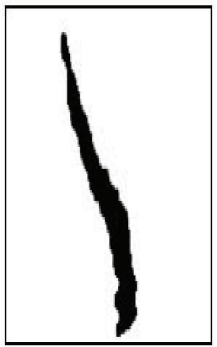

(c)

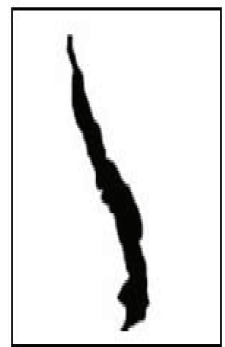

(d)

Fig. 3: Visual results of oil spill segmentation for Gaussian blurry NOWPAP SAR images: (a-1) Gaussian blurry oil spill images; (a-2) Straightforward segmentation results. (b-1) Deblurred images; (b-2) Oil spill segmentation of deblurred images (graph cuts); (b-3) Oil spill segmentation of deblurred images (DRLSE); (b-4) Oil spill segmentation of deblurred images (RSF); (c) ADMM segmentation results; (d) Ground truth. 

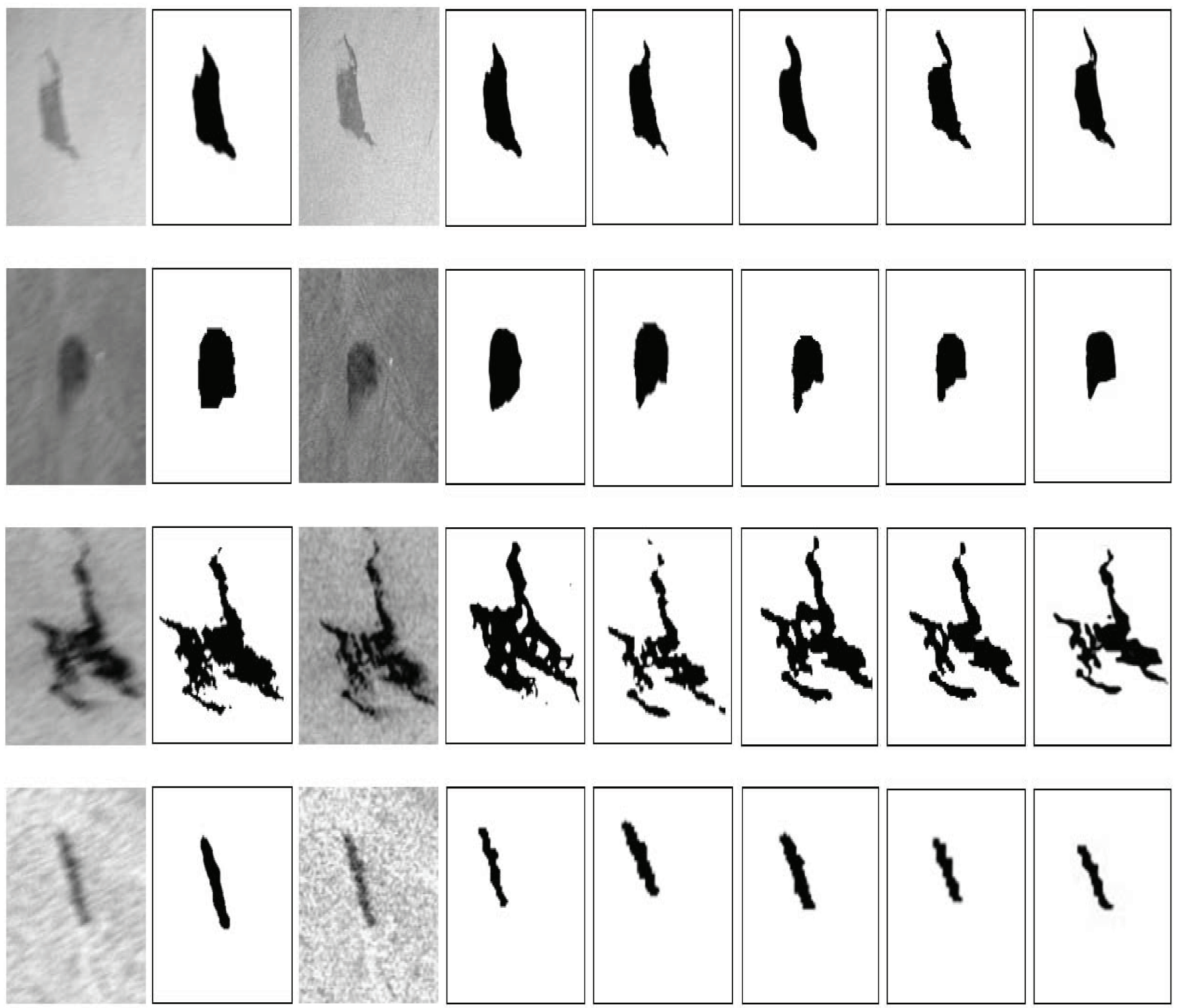
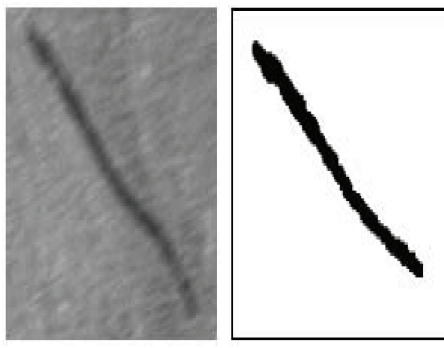

$(\mathrm{a}-2)$

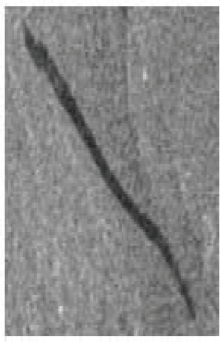

(b-1)

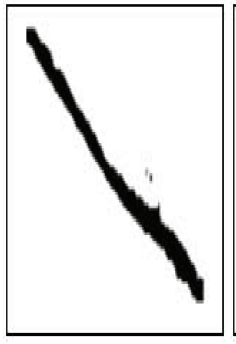

(b-2)

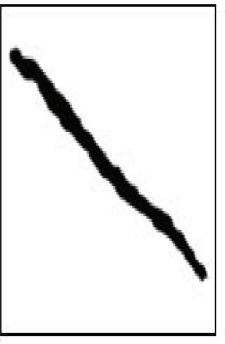

(b-3)

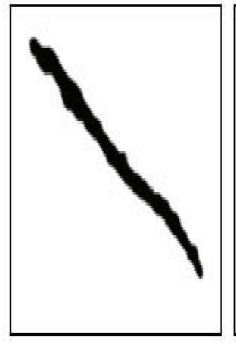

(b-4)

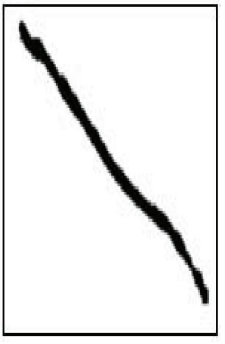

(c)

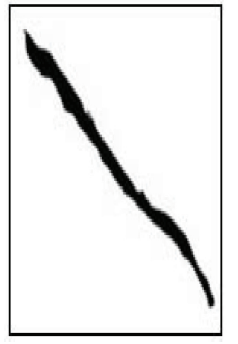

(d)

Fig. 4: Visual results of oil spill segmentation for motion blurry NOWPAP SAR images: (a-1) Motion blurry oil spill images; (a-2) Straightforward segmentation results. (b-1) Deblurred images; (b-2) Oil spill segmentation of deblurred images (graph cuts); (b-3) Oil spill segmentation of deblurred images (DRLSE); (b-4) Oil spill segmentation of deblurred images (RSF); (c) ADMM segmentation results; (d) Ground truth. 

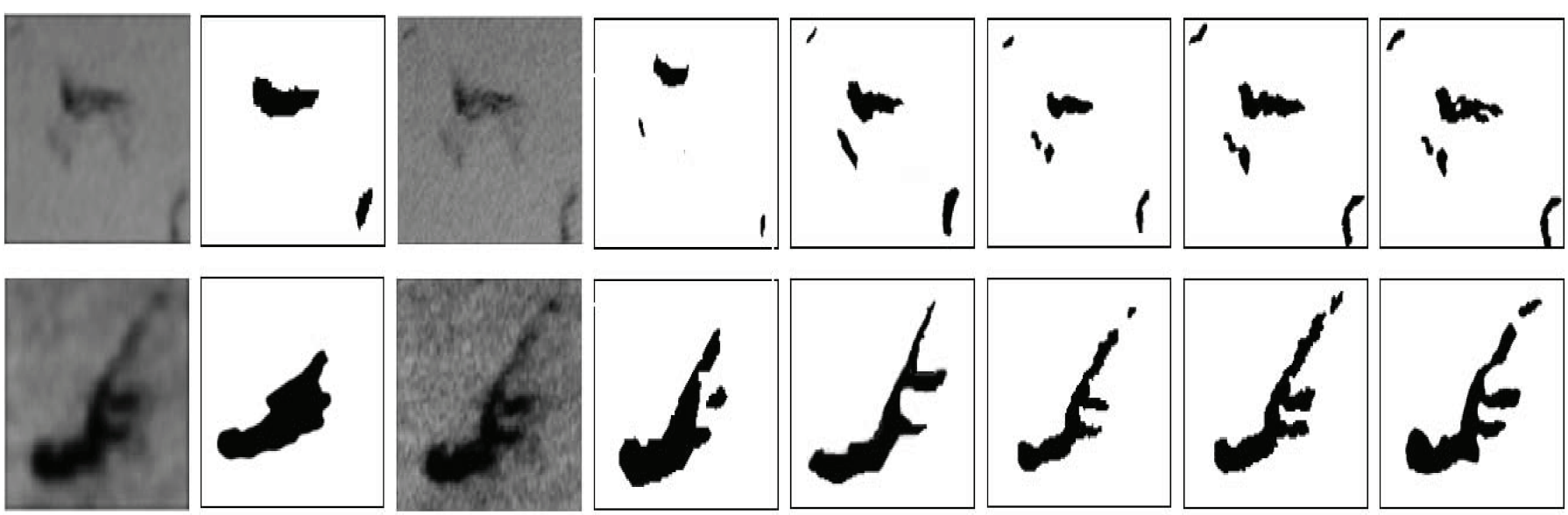

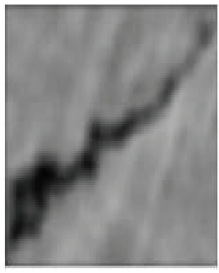

(a-1)

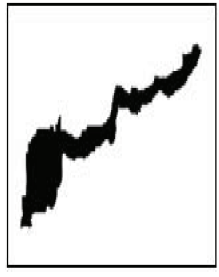

(a-2)

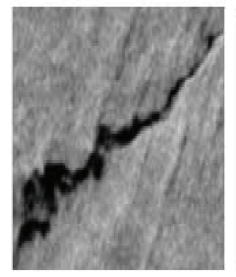

(b-1)

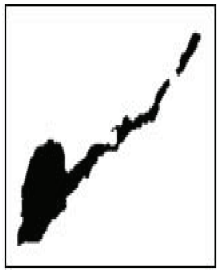

(b-2)

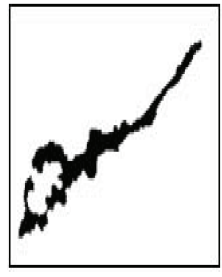

(b-3)

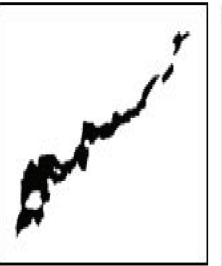

(b-4)

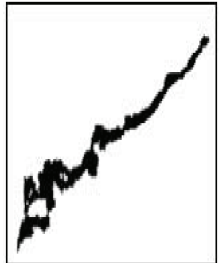

(c)

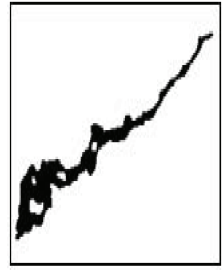

(d)

Fig. 5: Visual results of oil spill segmentation for Gaussian blurry UAVSAR images: (a-1) Gaussian blurry oil spill images; (a-2) Straightforward segmentation results. (b-1) Deblurred images; (b-2) Oil spill segmentation of deblurred images (graph cuts); (b-3) Oil spill segmentation of deblurred images (DRLSE); (b-4) Oil spill segmentation of deblurred images (RSF); (c) ADMM segmentation results; (d) Ground truth.
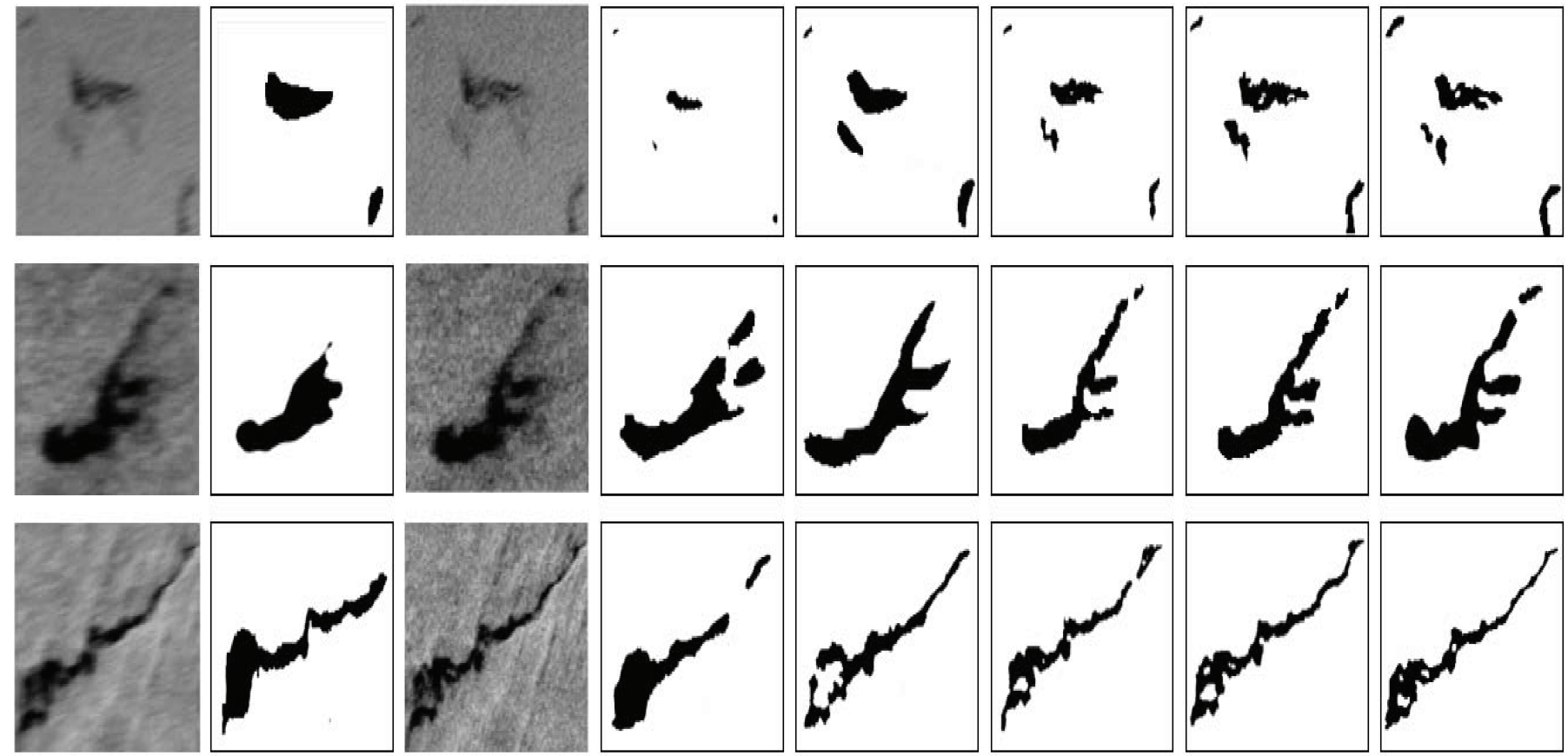

(a-1)

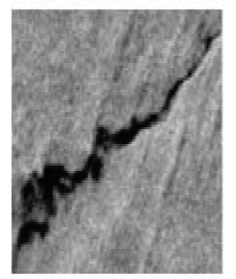

(b-1)

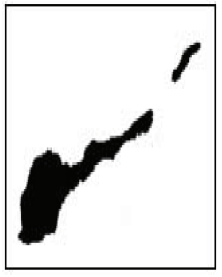

(b-2)
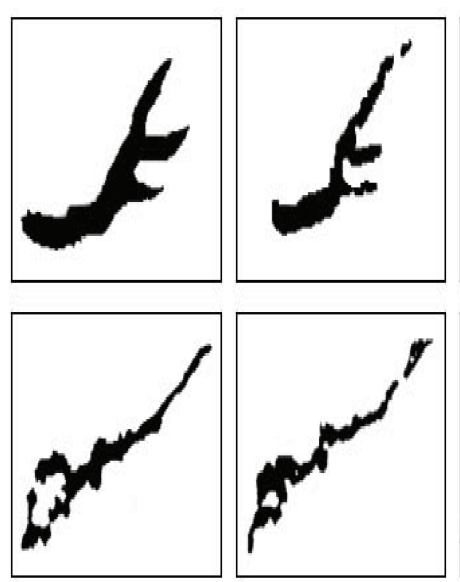

(b-3)

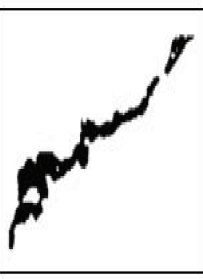

(b-4)
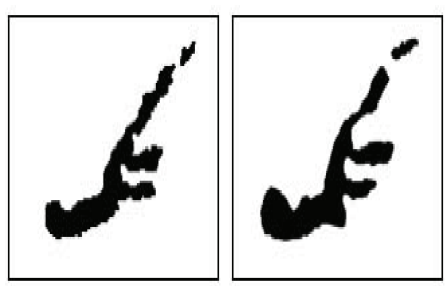

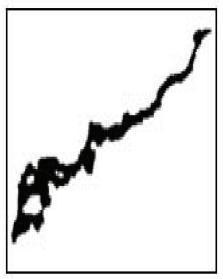

(c)

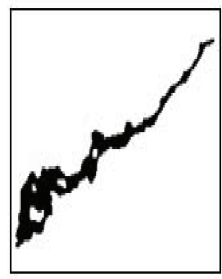

(d)

Fig. 6: Visual results of oil spill segmentation for motion blurry UAVSAR images: (a-1) Gaussian blurry oil spill images; (a-2) Straightforward segmentation results. (b-1) Deblurred images; (b-2) Oil spill segmentation of deblurred images (graph cuts); (b-3) Oil spill segmentation of deblurred images (DRLSE); (b-4) Oil spill segmentation of deblurred images (RSF); (c) ADMM segmentation results; (d) Ground truth. 

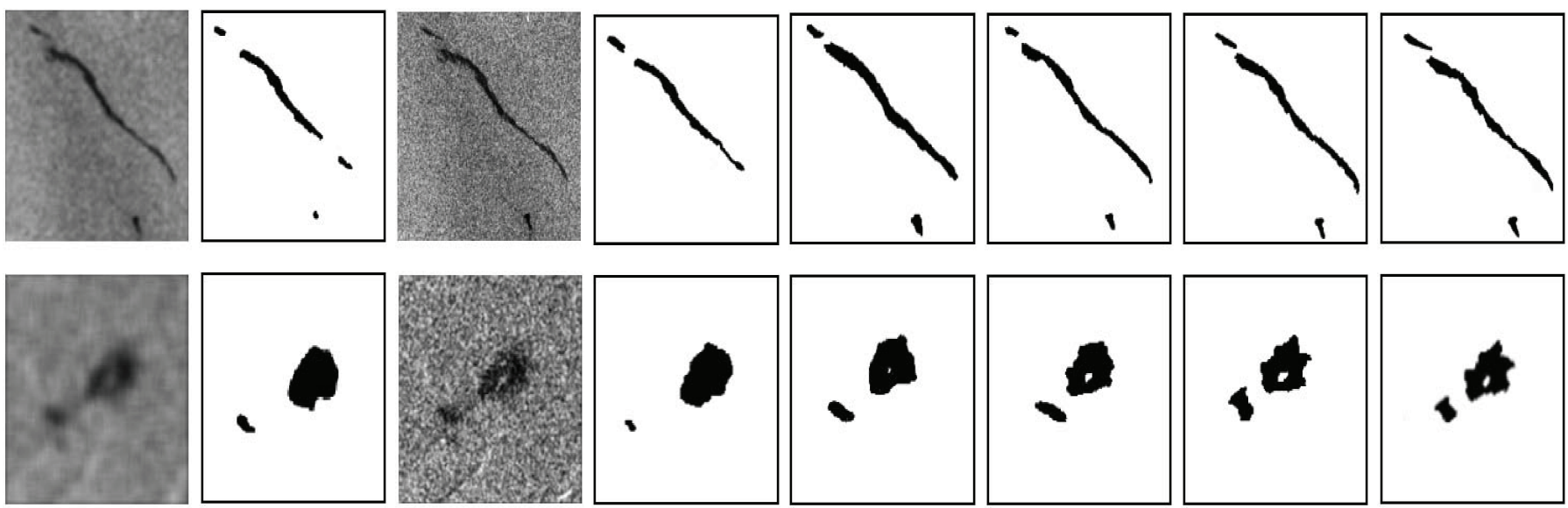

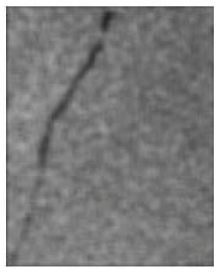

(a-1)

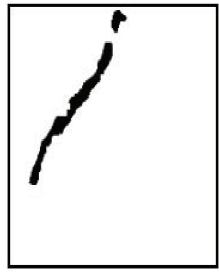

(a-2)

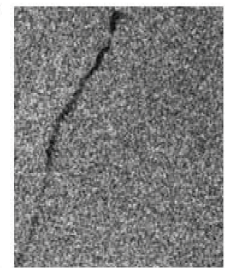

(b-1)

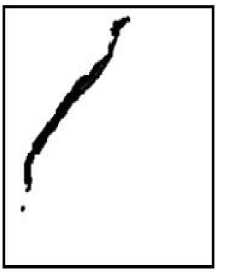

(b-2)

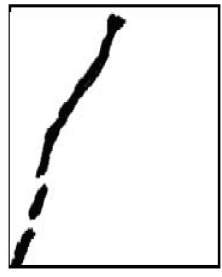

(b-3)

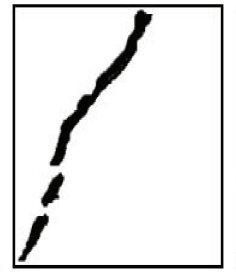

(b-4)

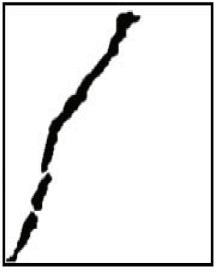

(c)

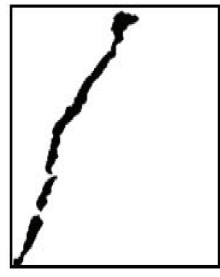

(d)

Fig. 7: Visual results of oil spill segmentation for Gaussian blurry MODIS images: (a-1) Gaussian blurry oil spill images; (a-2) Straightforward segmentation results. (b-1) Deblurred images; (b-2) Oil spill segmentation of deblurred images (graph cuts); (b-3) Oil spill segmentation of deblurred images (DRLSE); (b-4) Oil spill segmentation of deblurred images (RSF); (c) ADMM segmentation results; (d) Ground truth.
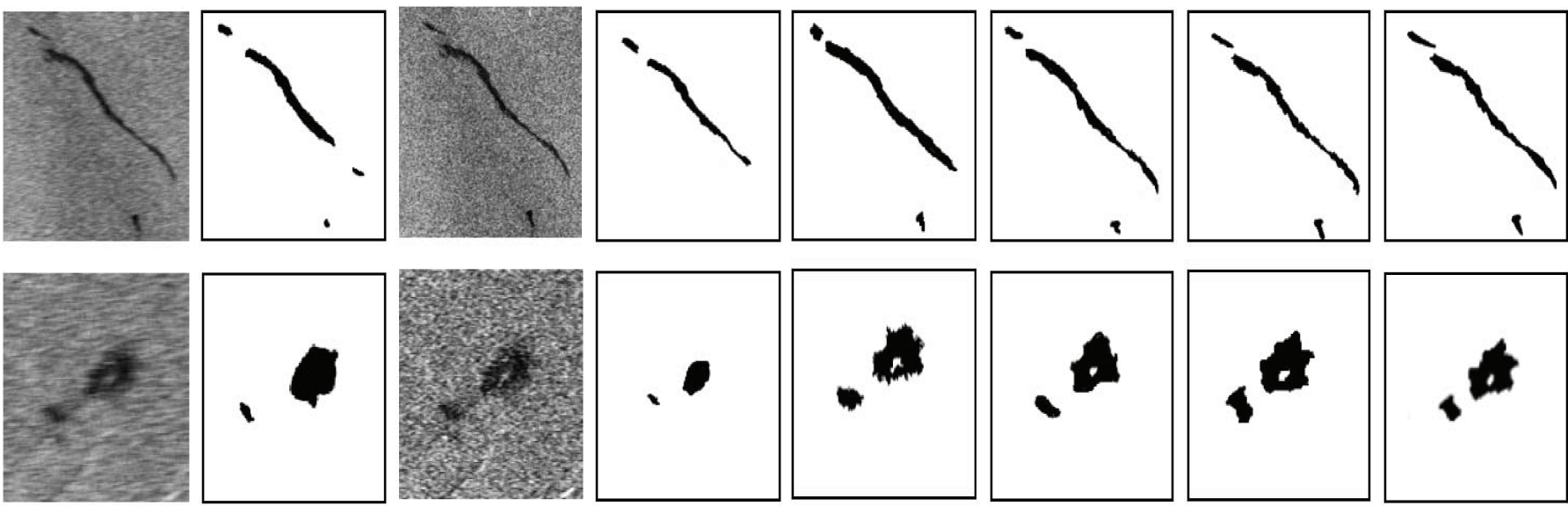

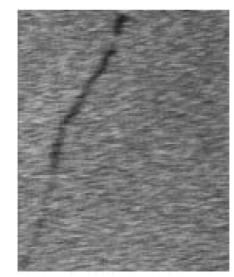

(a-1)

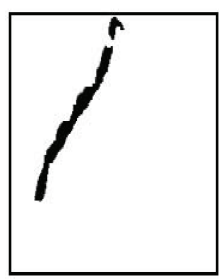

$(\mathrm{a}-2)$

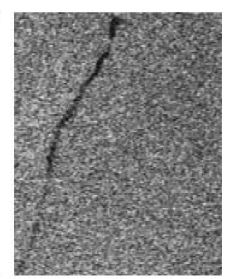

(b-1)

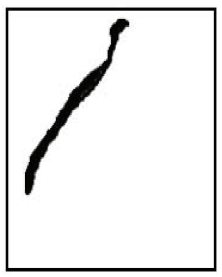

(b-2)

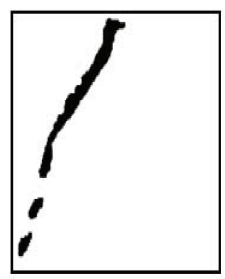

(b-3)

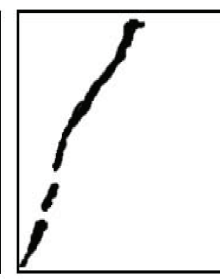

(b-4)

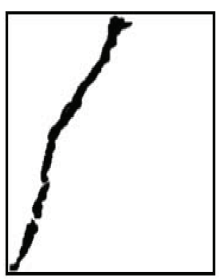

(c)

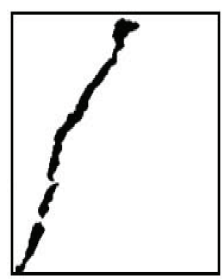

(d)

Fig. 8: Visual results of oil spill segmentation for motion blurry MODIS images: (a-1) Gaussian blurry oil spill images; (a-2) Straightforward segmentation results. (b-1) Deblurred images; (b-2) Oil spill segmentation of deblurred images (graph cuts); (b-3) Oil spill segmentation of deblurred images (DRLSE); (b-4) Oil spill segmentation of deblurred images (RSF); (c) ADMM segmentation results; (d) Ground truth. 

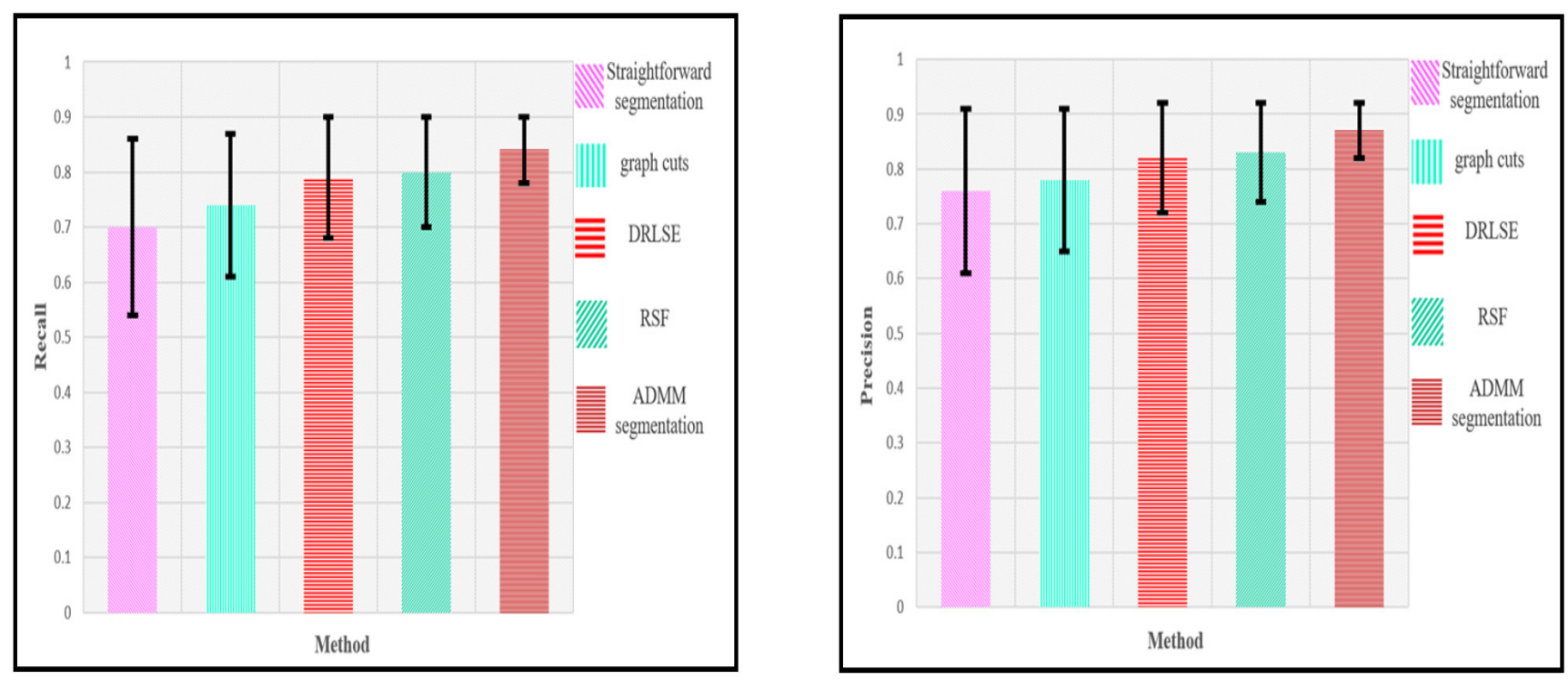

Fig. 9: Accuracy and standard deviation for Gaussian blurry oil spill image segmentation.
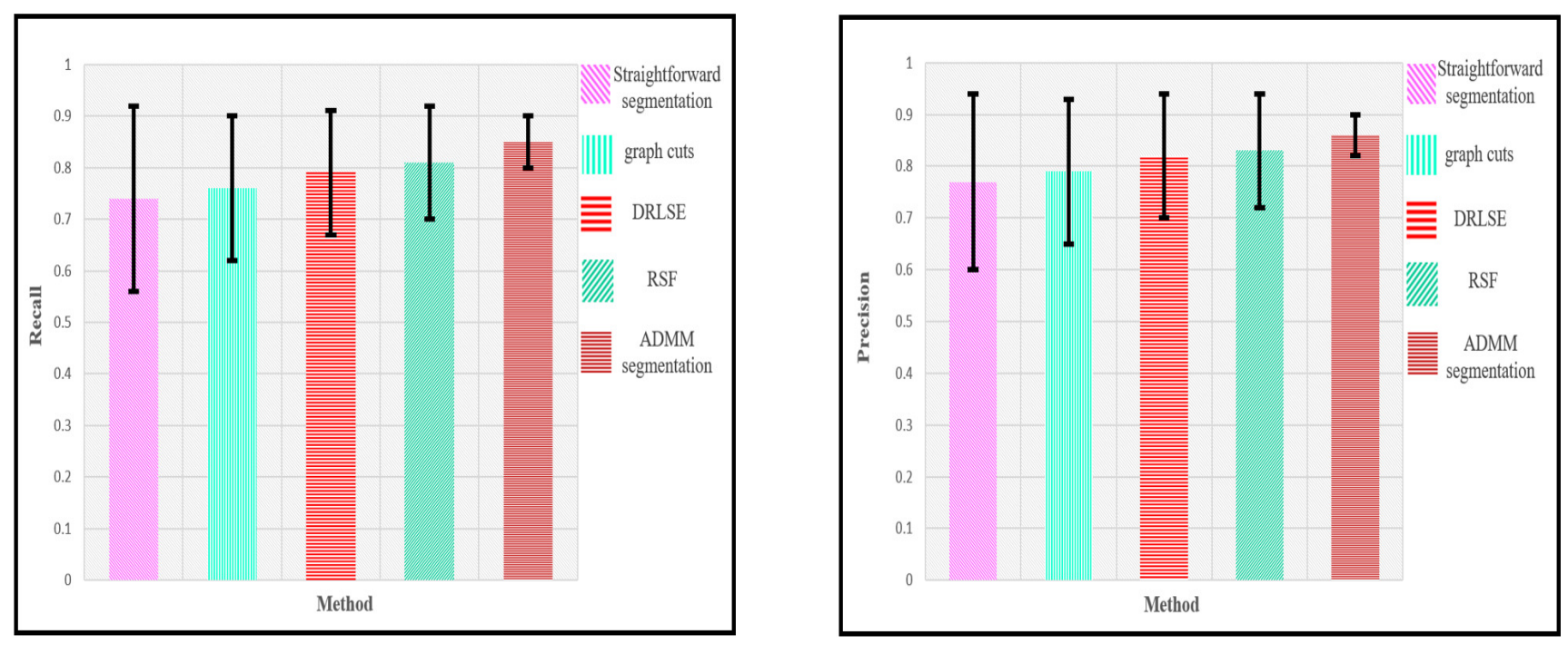

Fig. 10: Accuracy and standard deviation for motion blurry oil spill image segmentation. 

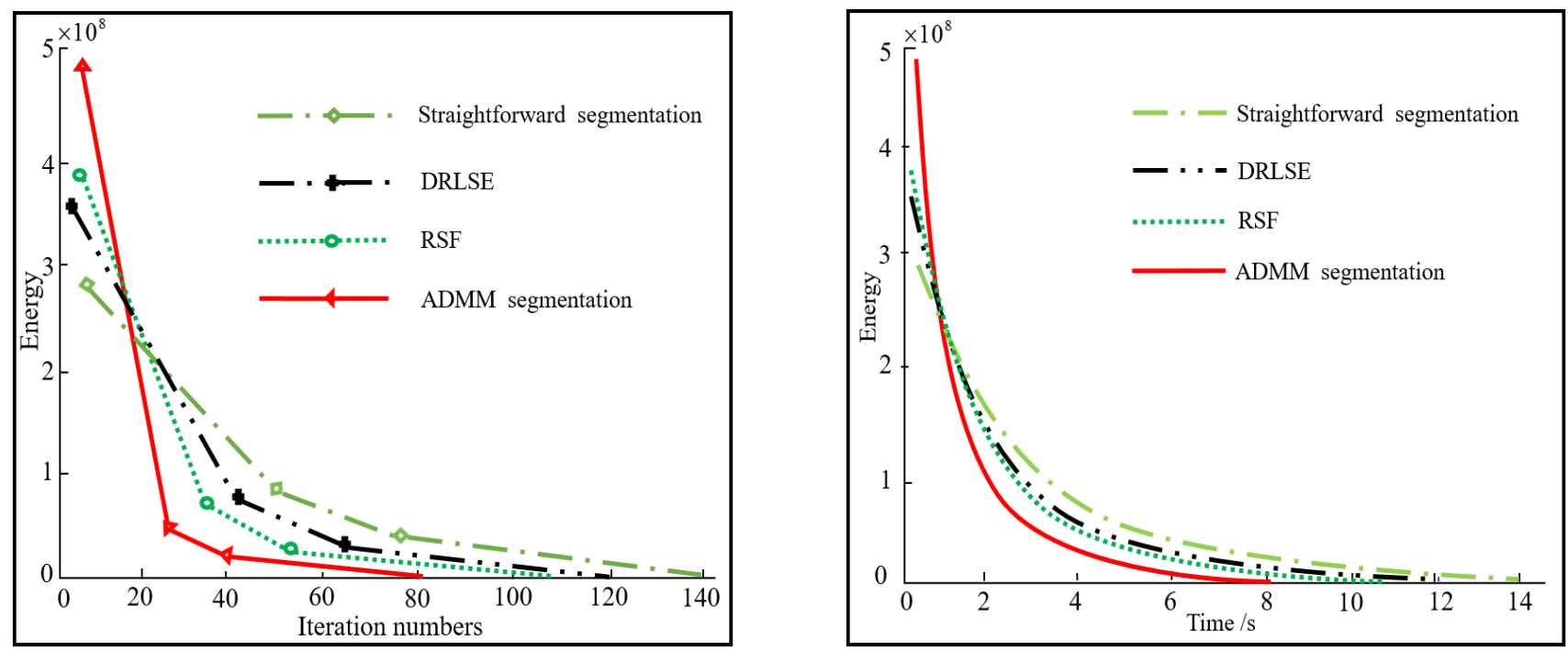

Fig. 11: Convergence rates with respect to the number of iterations. Fig. 12: Convergence rates with respect to runtime. 